\title{
A PARTIAL SOLUTION OF THE ISOPERIMETRIC PROBLEM FOR THE HEISENBERG GROUP
}

\author{
DONATELLA DANIELLI, NICOLA GAROFALO, AND DUY-MINH NHIEU
}

\section{Contents}

\begin{tabular}{|lr|}
\hline 1. Introduction & 1 \\
\hline 2. Isoperimetric inequalities in Carnot groups & 6 \\
\hline 3. Partial solution of the isoperimetric problem in $\mathbb{H}^{n}$ & 12 \\
\hline References & 30 \\
\hline
\end{tabular}

\section{Introduction}

The classical isoperimetric problem states that among all measurable sets with assigned volume the ball minimizes the perimeter. This is the content of the celebrated isoperimetric inequality, see DG3,

$$
|E|^{\frac{n-1}{n}} \leq C_{n} P(E)
$$

which holds for all measurable sets $E \subset \mathbb{R}^{n}$ with constant $C_{n}=n \sqrt{\pi} / \Gamma(n / 2+1)^{1 / n}$. In (1.1), $P(E)$ denotes the perimeter in the sense of De Giorgi, see DG1, DG2, i.e., the total variation of the indicator function of $E$. Equality holds in (1.1) if and only if (up to negligible sets) $E=B(x, R)=\left\{y \in \mathbb{R}^{n}|| y-x \mid<R\right\}$, a Euclidean ball. It is well-known that (1.1) is equivalent to the geometric Sobolev inequality for $B V$ functions, see [FR]. An analogous "isoperimetric inequality" was proved in GN] in the general setting of a Carnot-Carathéodory space, and such inequality was used, among other things, to establish a geometric embedding for horizontal $B V$ functions, similar to Fleming and Rishel's one. However, the question of the optimal configurations in such isoperimetric inequality was left open.

The aim of this paper is to bring a partial solution to this open problem in the Heisenberg group $\mathbb{H}^{n}$. We recall that $\mathbb{H}^{n}$ is the simplest and perhaps most important prototype of a class of nilpotent Lie groups, called Carnot groups, which play a fundamental role in analysis and geometry, see [Ca], Ch], [H], [St], Be], Gro1, Gro2, [E1], [E2], E3], DGN2]. Its underlying manifold is $\mathbb{R}^{2 n+1}$ with non-commutative group law

$$
g g^{\prime}=(x, y, t)\left(x^{\prime}, y^{\prime}, t^{\prime}\right)=\left(x+x^{\prime}, y+y^{\prime}, t+t^{\prime}+\frac{1}{2}\left(<x, y^{\prime}>-<x^{\prime}, y>\right)\right),
$$

Key words and phrases. Minimal surfaces, mean curvature, isoperimetric inequality, minimizers, best constant. First author supported in part by NSF grants DMS-0002801 and CAREER DMS-0239771.

Second author supported in part by NSF Grants DMS-0070492 and DMS-0300477. 
where we have let $x, x^{\prime}, y, y^{\prime} \in \mathbb{R}^{n}, t, t^{\prime} \in \mathbb{R}$. If $L_{g}\left(g^{\prime}\right)=g g^{\prime}$ denotes the operator of lefttranslation, let $\left(L_{g}\right)_{*}$ indicate its differential. The Heisenberg algebra admits the decomposition $\mathfrak{h}_{n}=V_{1} \oplus V_{2}$, where $V_{1}=\mathbb{R}^{2 n} \times\{0\}$, and $V_{2}=\{0\} \times \mathbb{R}$. Identifying $\mathfrak{h}_{n}$ with the space of left-invariant vector fields on $\mathbb{H}^{n}$, one easily recognizes that a basis for $\mathfrak{h}_{n}$ is given by the $2 n+1$ vector fields

$$
\left\{\begin{array}{l}
\left(L_{g}\right)_{*}\left(\frac{\partial}{\partial x_{i}}\right) \stackrel{\text { def }}{=} X_{i}=\frac{\partial}{\partial x_{i}}-\frac{y_{i}}{2} \frac{\partial}{\partial t}, \\
\left(L_{g}\right)_{*}\left(\frac{\partial}{\partial y_{i}}\right) \stackrel{\text { def }}{=} X_{n+i}=\frac{\partial}{\partial y_{i}}+\frac{x_{i}}{2} \frac{\partial}{\partial t}, \\
\left(L_{g}\right)_{*}\left(\frac{\partial}{\partial t}\right) \stackrel{\text { def }}{=} T=\frac{\partial}{\partial t},
\end{array}\right.
$$

and that the only non-trivial commutation relation is

$$
\left[X_{i}, X_{n+j}\right]=T \delta_{i j}, \quad i, j=1, \ldots, n .
$$

In (1.3) we have identified the standard basis $\left\{e_{1}, \ldots, e_{2 n}, e_{2 n+1}\right\}$ of $\mathbb{R}^{2 n+1}$ with the system of (constant) vector fields $\left\{\partial / \partial x_{1}, \ldots, \partial / \partial y_{n}, \partial / \partial t\right\}$. Because of (1.4) we have $\left[V_{1}, V_{1}\right]=V_{2}$, $\left[V_{1}, V_{2}\right]=\{0\}$, thus $\mathbb{H}^{n}$ is a graded nilpotent Lie group of step $r=2$. Lebesgue measure $d g=d z d t$ is a bi-invariant Haar measure on $\mathbb{H}^{n}$. If we denote by $\delta_{\lambda}(z, t)=\left(\lambda z, \lambda^{2} t\right)$ the nonisotropic dilations associated with the grading of the Lie algebra, then $d \circ \delta_{\lambda}=\lambda^{Q} d g$, where $Q=2 n+2$ is the homogeneous dimension of $\mathbb{H}^{n}$.

In what follows we denote by $P_{H}\left(E ; \mathbb{H}^{n}\right)$ the intrinsic, or $H$-perimeter of $E \subset \mathbb{H}^{n}$ associated with the bracket-generating system $X=\left\{X_{1}, \ldots, X_{2 n}\right\}$. Such notion will be recalled in Section 2 To state our theorem we let $\mathbb{H}_{+}^{n}=\left\{(z, t) \in \mathbb{H}^{n} \mid t>0\right\}, \mathbb{H}_{-}^{n}=\left\{(z, t) \in \mathbb{H}^{n} \mid t<0\right\}$, and consider the collection

$$
\mathcal{E}=\left\{E \subset \mathbb{H}^{n} \mid E \quad \text { satisfies }(i)-(i i)\right\}
$$

where

(i) $\left|E \cap \mathbb{H}_{+}^{n}\right|=\left|E \cap \mathbb{H}_{-}^{n}\right|$;

(ii) there exist $R>0$, and functions $u, v: \bar{B}(0, R) \rightarrow[0, \infty)$, with $u, v \in C^{1}(B(0, R)) \cap$ $C(\bar{B}(0, R)), u=v=0$ on $\partial B(0, R)$, and such that

$$
\begin{gathered}
\partial E \cap \mathbb{H}_{+}^{n}=\left\{(z, t) \in \mathbb{H}_{+}^{n}|| z \mid<R, t=u(z)\right\}, \\
\partial E \cap \mathbb{H}_{-}^{n}=\left\{(z, t) \in \mathbb{H}_{-}^{n}|| z \mid<R, t=-v(z)\right\} .
\end{gathered}
$$

We stress that the upper and lower portions of a set $E \in \mathcal{E}$ can be described by possibly different $C^{1}$ graphs, and that, besides $C^{1}$ smoothness, and the fact that their common domain is a ball, no additional assumption is made on the functions $u$ and $v$. For instance, we do not require a priori that $u$ and/or $v$ are spherically symmetric. Here is our main result.

Theorem 1.1. Let $V>0$, and define the number $R>0$ by

$$
R=\left(\frac{(Q-2) \Gamma\left(\frac{Q+2}{2}\right) \Gamma\left(\frac{Q-2}{2}\right)}{\pi^{\frac{Q-1}{2}} \Gamma\left(\frac{Q+1}{2}\right)}\right)^{1 / Q} V^{1 / Q} .
$$

Given such $R$, then the variational problem

$$
\min _{E \in \mathcal{E},|E|=V} P_{H}\left(E ; \mathbb{H}^{n}\right)
$$


has a unique solution $E_{R}=\delta_{R}\left(E_{o}\right) \in \mathcal{E}$, where $\partial E_{o}$ is described by the graph $t= \pm u_{o}(z)$, with

$$
u_{o}(z) \stackrel{\text { def }}{=}\left\{\frac{\pi}{8}+\frac{|z|}{4} \sqrt{1-|z|^{2}}-\frac{1}{4} \sin ^{-1}(|z|)\right\}, \quad|z| \leq 1 .
$$

The sign \pm depends on whether one considers $\partial E_{o} \cap \mathbb{H}_{+}^{n}$, or $\partial E_{o} \cap \mathbb{H}_{-}^{n}$. Finally, the boundary $\partial E_{R}=\delta_{R}\left(\partial E_{o}\right)$ of the bounded open set $E_{R}$ is only of class $C^{2}$, but not of class $C^{3}$, near its two characteristic points $\left(0, \pm \frac{\pi R^{2}}{8}\right)$, it is $C^{\infty}$ away from them, and $S_{R}=\partial E_{R}$ has positive constant $H$-mean curvature given by

$$
\mathcal{H}=\frac{Q-2}{R} .
$$

For the notion of $H$-mean curvature of a $C^{2}$ hypersurface $\mathcal{S} \subset \mathbb{H}^{n}$ we refer the reader to (3.11) in Section 3. This notion of horizontal mean curvature, which is of course central to the present study, was introduced in DGN4. Its geometric interpretation is that, in the neighborhood of a non-characteristic point $g \in \mathcal{S}$, it coincides with the standard Riemannian mean curvature of the $2 n$-1-dimensional submersed manifold obtained by intersecting the hypersurface $\mathcal{S}$ with the fiber of the horizontal subbundle $H_{g} \mathbb{H}^{n}$, see also [DGN3] where a related notion of Gaussian curvature was introduced. A seemingly different notion, based on the Riemannian regularization of the sub-Riemannian metric of $\mathbb{H}^{n}$, was proposed in $[\mathrm{Pa}]$, but the two are in fact equivalent, see DGN4. From Theorem 1.1] we obtain the following isoperimetric inequality for the horizontal perimeter.

Theorem 1.2. Let $\mathcal{E}$ be as above, and denote by $\tilde{\mathcal{E}}$ the class of sets of the type $\delta_{\lambda} L_{g}(E)$, for some $E \in \mathcal{E}, \lambda>0$ and $g \in \mathbb{H}^{n}$, then the following isoperimetric inequality holds

$$
|E|^{\frac{Q-1}{Q}} \leq C_{Q} P(E), \quad E \in \tilde{\mathcal{E}},
$$

where

$$
C_{Q}=\frac{(Q-1) \Gamma\left(\frac{Q}{2}\right)^{\frac{2}{Q}}}{Q^{\frac{Q-1}{Q}}(Q-2) \Gamma\left(\frac{Q+1}{2}\right)^{\frac{1}{Q}} \pi^{\frac{Q-1}{2 Q}}},
$$

with equality if and only if for some $\lambda>0$ and $g \in \mathbb{H}^{n}$ one has $E=L_{g} \delta_{\lambda}\left(E_{o}\right)$, where $E_{o}$ is given by (1.5).

Fig.1.1 gives a representation of the isoperimetric set $E_{o}$ in Theorem 1.1 in the special case $n=1$. We note that the invariance of the isoperimetric quotient with respect to the group left-translations $L_{g}$ and dilations $\delta_{\lambda}$ is guaranteed by Propositions 2.11 and 2.12 ,

A remarkable property of the isoperimetric sets is that, similarly to their Riemannian predecessors, they have constant $H$-mean curvature. It is tempting, and also natural, to conjecture that the set $E_{o}$ described by (1.5), along with its left-translated and dilated, exaust all the isoperimetric sets in $\mathbb{H}^{n}$ (for the definition of such sets, see Definition 1.4 below). By this we mean that Theorem 1.2 continues to be valid when one replaces the class $\tilde{\mathcal{E}}$ with that of all measurable sets $E \subset \mathbb{H}^{n}$ with locally finite $H$-perimeter. At the moment, this remains a challenging open 


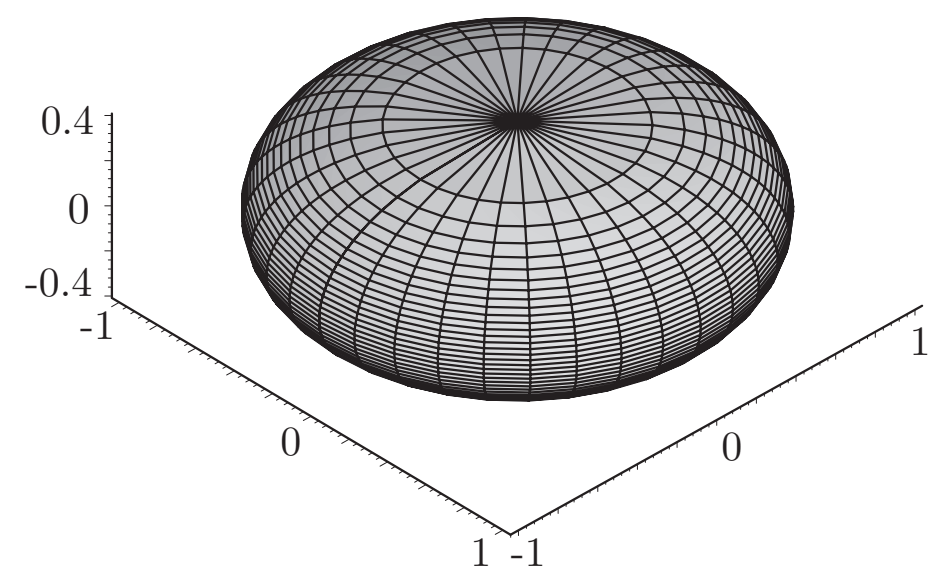

FiguRE 1.1. Isoperimetric set in $\mathbb{H}^{1}$ with $R=1$

problem. In this connection, another interesting conjecture is as follows: Let $\mathcal{S} \subset \mathbb{H}^{n}$ be a $C^{2}$, compact oriented hypersurface. Suppose that for some $\alpha>0$

$$
\mathcal{H} \equiv \alpha \quad \text { on } \mathcal{S} .
$$

Is it true that, if we denote by $\mathcal{S}^{+}=\mathcal{S} \cap \mathbb{H}_{+}^{n}, \mathcal{S}^{-}=\mathcal{S} \cap \mathbb{H}_{-}^{n}$, then $\mathcal{S}^{+}, \mathcal{S}^{-}$are respectively described by

$$
t= \pm\left\{\frac{1}{4}|z| \sqrt{R^{2}-|z|^{2}}-\frac{R^{2}}{4} \tan ^{-1}\left(\frac{|z|}{\sqrt{R^{2}-|z|^{2}}}\right)+\frac{\pi R^{2}}{8}\right\}, \quad|z| \leq R,
$$

where $R=(Q-2) / \alpha$ ? Concerning this conjecture we remark that Theorem 1.1provides evidence in favor of it. As it is well-known, the Euclidean counterpart of it is contained in the celebrated soap bubble theorem of A.D. Alexandrov $[$ ].

To put the above results in a broader perspective we recall that in any Carnot group a general scale invariant isoperimetric inequality is available. In fact, using the results in [CDG], GN] one can prove the following theorem, see Theorem 2.9] in Section 2

Theorem 1.3. Let $\boldsymbol{G}$ be a Carnot group with homogeneous dimension $Q$. There exists a constant $C_{\text {iso }}(\boldsymbol{G})>0$ such that, for every $H$-Caccioppoli set $E \subset \boldsymbol{G}$, one has

$$
|E|^{(Q-1) / Q} \leq C_{i s o}(\boldsymbol{G}) P_{H}(E ; \boldsymbol{G}) .
$$

A measurable set $E \subset \boldsymbol{G}$ is called a $H$-Caccioppoli set if $P_{H}(E ; \omega)<\infty$ for any $\omega \subset \subset \boldsymbol{G}$. Theorem 1.3 generalizes an earlier result of Pansu [P1], who proved a related inequality for the first Heisenberg group $\mathbb{H}^{1}$, but with the $H$-perimeter in the right-hand side replaced by the 3-dimensional Hausdorff measure $\mathcal{H}^{3}$ in $\mathbb{H}^{1}$ constructed with the Carnot-Carathéodory distance associated with the horizontal subbundle $H \mathbb{H}^{1}$ defined by $\left\{X_{1}, X_{2}\right\}$ in (1.3). One should keep in mind that the homogeneous dimension of $\mathbb{H}^{1}$ is $Q=4$, so $3=Q-1$, which explains the appearance of $\mathcal{H}^{3}$ in Pansu's result. It should also be said that some authors attribute to Pansu [P2] the conjecture that the isoperimetric sets in $\mathbb{H}^{1}$ have the form (1.5). We mention that other isoperimetric and Fleming-Rishel type Gagliardo-Nirenberg inequalities have been obtained by 
several authors at several times, see [Va1, Va2, [VSC, CS, BM], FGW], MaSC]. We now introduce the following definition.

Definition 1.4. Given a Carnot group $\boldsymbol{G}$ with homogeneous dimension $Q$ we define the isoperimetric constant of $\boldsymbol{G}$ as

$$
\alpha_{i s o}(\boldsymbol{G})=\inf _{E \subset \boldsymbol{G}} \frac{P_{H}(E ; \boldsymbol{G})}{|E|^{(Q-1) / Q}},
$$

where the infimum is taken on all $H$-Caccioppoli sets $E$ such that $0<|E|<\infty$. If a measurable set $E_{o}$ is such that

$$
\alpha_{i s o}(\boldsymbol{G})=\frac{P_{H}\left(E_{o} ; \boldsymbol{G}\right)}{\left|E_{o}\right|^{(Q-1) / Q}},
$$

then we call it an isoperimetric set in $\boldsymbol{G}$.

We stress that, thanks to Theorem 1.3, the isoperimetric constant is strictly positive. It should also be observed that, using the representation formula for the $H$-perimeter

$$
P_{H}(E ; \boldsymbol{G})=\int_{\partial E} \frac{W}{|\boldsymbol{N}|} d H_{N-1}
$$

valid for any bounded open set $E \subset \boldsymbol{G}$ of class $C^{1}$, with Riemannian outer normal $\boldsymbol{N}$ and angle function $W=\sqrt{p_{1}^{2}+\ldots+p_{m}^{2}}$ (see Lemma [2.8 and (3.1), (3.2)), one immediately recognizes that, since for any $\omega \subset \subset \boldsymbol{G}$ one has $W \leq C(\omega)|\boldsymbol{N}|$, then $P_{H}(E ; \boldsymbol{G}) \leq C H_{N-1}(\partial E)<\infty$. As a consequence, $\alpha_{i s o}(\boldsymbol{G})<\infty$ as well. What is not obvious instead is the existence of isoperimetric sets. In this regard, one has the following basic result proved in [LR].

Theorem 1.5. Let $\boldsymbol{G}$ be a Carnot group, then there exists a bounded $H$-Caccioppoli set $F_{o}$ such that

$$
P_{H}\left(F_{o} ; \boldsymbol{G}\right)=\alpha_{i s o}(\boldsymbol{G})\left|F_{o}\right|^{(Q-1) / Q} .
$$

The equality continues to be valid if one replaces $F_{o}$ by $L_{g_{o}} \circ \delta_{\lambda}\left(F_{o}\right)$, for any $\lambda>0, g_{o} \in \boldsymbol{G}$.

Of course, this result leaves open the fundamental question of the classification of such sets. We stress that, in the generality of Theorem 1.3. this problem is presently totally out of reach. When $\boldsymbol{G}=\mathbb{H}^{n}$, however, Theorems 1.1 and 1.2 provide some basic progress in this direction.

In connection with our work, we mention the interesting recent work $[\mathrm{BC}]$ in which, for the first Heisenberg group $\mathbb{H}^{1}$, the authors prove that the flow by $H$-mean curvature of a $C^{2}$ surface which is convex, and which is described by $t= \pm f(|z|)$, with $f^{\prime}>0$, converges to the isoperimetric sets (1.5). Notice, however, that $f \in C^{2}$, spherically symmetric, convex, and that it is assumed that the upper and lower part of the surface are described by the same strictly decreasing function $f$. We also mention the paper $[\mathrm{Pa}]$ in which the author, still for $\mathbb{H}^{1}$, heuristically derives the surface described by (1.5) by imposing the condition of constant $H$-mean curvature among all $C^{2}$ surfaces which can be described by $t= \pm f(|z|)$. Recently, Hladky and Pauls in [HP] have proposed a general geometric framework, which they call the class of Vertically Rigid manifolds, and which encompasses the class of Carnot groups, in which they study the isoperimetric and the minimal surface problems. In this setting they introduce a notion of horizontal mean curvature, and they show, in particular, that remarkably the isoperimetric sets have constant horizontal 
mean curvature. In the paper [LM] the authors prove, among other interesting results, that the $u_{o}$ in our Theorem 1.1 is a critical point (but not the unique minimizer) of the $H$-perimeter, when the class of competitors is restricted to $C^{2}$ domains, with defining function of the type $t= \pm f(|z|)$. A similar result has been also obtained in the interesting recent preprint [RR], which also contains a classification of the Delaunay type surfaces in $\mathbb{H}^{n}$. In this connection, we also mention the earlier paper [To, in which the author describes the Delaunay type surfaces of revolution in $\mathbb{H}^{1}$, heuristically computes the special solutions (1.5), and shows that standard Schwarz symmetrization does not work in the Heisenberg group. In FMP the authors gave a complete classification of the constant mean curvature surfaces (including minimal) which are invariant with respect to 1-dimensional closed subgroups of $\operatorname{Iso}_{0}\left(H_{3}, g\right)$. We also mention the paper Mo1, in which the author proved that the Carnot-Carathéodory ball in $\mathbb{H}^{n}$ is not an isoperimetric set. Subsequently, in [Mo2] he proved that, as a consequence of this fact, a generalization of the Brunn-Minkowski inequality to $\mathbb{H}^{n}$ fails. Finally, in their interesting paper MoM] the authors have established an isoperimetric inequality for the Baouendi-Grushin vector fields $X_{1}=\partial_{x}, X_{2}=|x|^{\alpha} \partial_{t}, \alpha>0$, in the plane $(x, t)$, and explicitly computed the isoperimetric profiles. In the special case $\alpha=1$, such profiles are identical (up to a normalization of the vector fields) to our $u_{o}$ in Theorem 1.1.

Acknowledgment $^{1}$ : For the first Heisenberg group $\mathbb{H}^{1}$, and under the assumption that the isoperimetric profile be of class $C^{2}$ and of the type $t=f(|z|)$, the idea of using calculus of variations to explicitly determine $f(|z|)$, first came about in computations that Giorgio Talenti and the second named author carried in a set of unpublished notes in Oberwolfach in 1995. We would like to thank G. Talenti for his initial contribution to the present study.

\section{Isoperimetric inequalities in Carnot groups}

The appropriateness of the notion of $H$-perimeter in Carnot-Carathéodory geometry is witnessed by the isoperimetric inequalities. Similarly to their Euclidean counterpart, these inequalities play a fundamental role in the development of geometric measure theory. Theorem 1.3 represents a sub-Riemannian analogue of the classical global isoperimetric inequality. Such result can be extracted from the isoperimetric inequalities obtained in [CDG] and [GN], but it is not explicitly stated in either paper. Since a proof of Theorem 1.3 is not readily available in the literature, for completeness we present it in this section.

Given a Carnot group $\boldsymbol{G}$, its Lie algebra $\mathfrak{g}$ satisfies the properties $\mathfrak{g}=V_{1} \oplus \ldots \oplus V_{r}$, where $\left[V_{1}, V_{j}\right]=V_{j+1}, j=1, \ldots, r-1$, and $\left[V_{1}, V_{r}\right]=\{0\}$. If $m_{j}=\operatorname{dim} V_{j}, j=1, \ldots, r$, then the

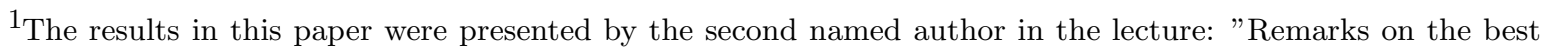
constant in the isoperimetric inequality for the Heisenberg group and surfaces of constant mean curvature", Analysis seminar, University of Arkansas, April 12, 2001, (http://comp.uark.edu/ lanzani/schedule.html), by the third named author at the international meeting on "Subelliptic equations and sub-Riemannian geometry", Arkansas, March 2003, and by the second named author in the lecture "Hypersurfaces of minimal type in subRiemannian geometry", Seventh New Mexico Analysis Seminar, University of New Mexico, October 2004.
} 
homogeneous dimension of $\boldsymbol{G}$ is defined by $Q=m_{1}+2 m_{2}+\ldots+r m_{r}$. The non-isotropic dilations associated with the grading of $\mathfrak{g}$ are given by $\Delta_{\lambda}\left(\xi_{1}+\ldots+\xi_{r}\right)=\lambda \xi_{1}+\ldots+\lambda^{r} \xi_{r}$. Via the exponential mapping exp : $\mathfrak{g} \rightarrow \boldsymbol{G}$, which is a global diffeomorphism onto, such dilations induce a one-parameter group of dilations on $\boldsymbol{G}$ as follows $\delta_{\lambda}(g)=\Delta_{\lambda}\left(\exp ^{-1} g\right)$. The push forward through exp of the standard Lebesgue measure on $\mathfrak{g}$ is a bi-invariant Haar measure on $\boldsymbol{G}$. We will denote it by $d g$. Clearly, $d\left(\delta_{\lambda} g\right)=\lambda^{Q} d g$. For simplicity, we let $m=m_{1}$. We fix orthonormal basis $\left\{e_{1}, \ldots, e_{m}\right\}, \ldots,\left\{e_{r, 1}, \ldots, e_{r, m_{r}}\right\}$, of the layers $V_{1}, \ldots, V_{r}$, and consider the corresponding left-invariant vector fields on $\boldsymbol{G}$ defined by $X_{1}(g)=\left(L_{g}\right)_{*}\left(e_{1}\right), \ldots, X_{m}(g)=\left(L_{g}\right)_{*}\left(e_{m}\right), \ldots$, $X_{r, 1}(g)=\left(L_{g}\right)_{*}\left(e_{r, 1}\right), \ldots, X_{r, m_{r}}(g)=\left(L_{g}\right)_{*}\left(e_{r, m_{r}}\right)$. We will assume that $\boldsymbol{G}$ is endowed with a left-invariant Riemannian metric $\langle\cdot, \cdot\rangle$ with respect to which these vector fields constitute and orthonormal basis. No other inner product will be used in this paper. We denote by $H \boldsymbol{G} \subset T \boldsymbol{G}$ the subbundle of the tangent bundle generated by $\left\{X_{1}, \ldots, X_{m}\right\}$. We next recall the notion of $H$-perimeter, see e.g. [CDG]. Given an open set $\Omega \subset G$, we let

$$
\mathcal{F}(\Omega)=\left\{\zeta=\left.\sum_{i=1}^{m} \zeta_{i} X_{i} \in \Gamma_{0}^{1}(\Omega, H \boldsymbol{G})|| \zeta\right|_{\infty}=\sup _{\Omega}|\zeta|=\sup _{\Omega}\left(\sum_{i=1}^{m} \zeta_{i}^{2}\right)^{1 / 2} \leq 1\right\},
$$

where we say that $\zeta \in \Gamma_{0}^{1}(\Omega, H \boldsymbol{G})$ if $X_{j} \zeta_{i} \in C_{0}(\Omega)$ for $i, j=1, \ldots, m$. Given $\zeta \in \Gamma_{0}^{1}(\Omega, H \boldsymbol{G})$ we define

$$
\operatorname{div}_{H} \zeta=\sum_{i=1}^{m} X_{i} \zeta_{i}
$$

For a function $u \in L_{l o c}^{1}(\Omega)$, the $H$-variation of $u$ with respect to $\Omega$ is defined by

$$
\operatorname{Var}_{H}(u ; \Omega)=\sup _{\zeta \in \mathcal{F}(\Omega)} \int_{G} u d i v_{H} \zeta d g .
$$

We say that $u \in L^{1}(\Omega)$ has bounded $H$-variation in $\Omega$ if $\operatorname{Var}_{H}(u ; \Omega)<\infty$. The space $B V_{H}(\Omega)$ of functions with bounded $H$-variation in $\Omega$, endowed with the norm

$$
\|u\|_{B V_{H}(\Omega)}=\|u\|_{L^{1}(\Omega)}+\operatorname{Var}_{H}(u ; \Omega),
$$

is a Banach space. A fundamental property of the space $B V_{H}$ is the following special case of the compactness Theorem 1.28 proved in [GN].

Theorem 2.1. Let $\Omega \subset \boldsymbol{G}$ be a $(P S)$ (Poincaré-Sobolev) domain. The embedding

$$
i: B V_{H}(\Omega) \hookrightarrow L^{q}(\Omega)
$$

is compact for any $1 \leq q<Q /(Q-1)$.

We now recall a special case of Theorem 1.4 in [CDG].

Theorem 2.2. Let $\boldsymbol{G}$ be a Carnot group with homogeneous dimension $Q$. There exists a constant $C(\boldsymbol{G})>0$, such that for every $g_{o} \in \boldsymbol{G}, 0<R<R_{o}$, one has for every $C^{1}$ domain $E \subset \bar{E} \subset$ $B\left(g_{o}, R\right)$

$$
|E|^{(Q-1) / Q} \leq C P_{H}\left(E ; B\left(g_{o}, R\right)\right) .
$$


To prove Theorem 1.3 we need to extend Theorem 2.2 from bounded $C^{1}$ domains to arbitrary sets having locally finite $H$-perimeter. That such extension be possible is due in part to the following approximation result for functions in the space $B V_{H}$, which is contained in Theorem 1.14 in [GN], see also [FSS1].

Theorem 2.3. Let $\Omega \subset \boldsymbol{G}$ be open, where $\boldsymbol{G}$ is a Carnot group. For every $u \in B V_{H}(\Omega)$ there exists a sequence $\left\{u_{k}\right\}_{k \in N}$ in $C^{\infty}(\Omega)$ such that

$$
\begin{gathered}
u_{k} \rightarrow u \quad \text { in } L^{1}(\Omega) \quad \text { as } k \rightarrow \infty, \\
\lim _{k \rightarrow \infty} \operatorname{Var}_{H}\left(u_{k} ; \Omega\right)=\operatorname{Var}_{H}(u ; \Omega) .
\end{gathered}
$$

We next introduce the notion of $H$-perimeter.

Definition 2.4. Let $E \subset G$ be a measurable set, $\Omega$ be an open set. The $H$-perimeter of $E$ with respect to $\Omega$ is defined by

$$
P_{H}(E ; \Omega)=\operatorname{Var}_{H}\left(\chi_{E} ; \Omega\right),
$$

where $\chi_{E}$ denotes the indicator function of $E$. We say that $E$ is a $H$-Caccioppoli set if $\chi_{E} \in$ $B V_{H}(\Omega)$ for every $\Omega \subset \subset \boldsymbol{G}$.

The reader will notice that when the step of the group $\boldsymbol{G}$ is $r=1$, and therefore $\boldsymbol{G}$ is Abelian, the space $B V_{H}$ coincides with the space $B V$ introduced by De Giorgi, see [DG1, DG2], DCP], and thereby in such setting the Definition 2.4 coincides with his notion of perimeter. A fundamental rectifiability theorem á la De Giorgi for $H$-Caccioppoli sets has been established, first for the Heseinberg group $\mathbb{H}^{n}$, and then for every Carnot group of step $r=2$, in the papers [FSS2, FSS3, FSS4]. We will need the following simple fact.

Lemma 2.5. Let $R_{o}>0$ be given and consider a $H$-Caccioppoli set $E \subset B\left(e, R_{o}\right)$, then

$$
P_{H}\left(E, B\left(e, R_{o}\right)\right)=P_{H}(E, \boldsymbol{G}) .
$$

Proof. This can be easily seen as follows. Clearly, one has trivially $P_{H}\left(E, B\left(e, R_{o}\right)\right) \leq P_{H}(E, G)$. To establish the opposite inequality, let $r_{o}<R_{o}$ be such that $E \subset B\left(e, r_{o}\right)$, and pick $f \in$ $C_{0}^{\infty}\left(B\left(e, R_{o}\right)\right)$ be such that $0 \leq f \leq 1$, and $f \equiv 1$ on $\left.\bar{B}\left(e, r_{o}\right)\right)$. If $\zeta \in \mathcal{F}(\boldsymbol{G})$, then it is clear that $f \zeta \in \Gamma_{0}^{1}\left(B\left(e, R_{o}\right) ; H \boldsymbol{G}\right)$, and that $\|f \zeta\|_{L^{\infty}\left(B\left(e, R_{o}\right)\right)} \leq 1$, i.e., $f \zeta \in \mathcal{F}\left(B\left(e, R_{o}\right)\right)$. We have

$$
\begin{aligned}
& \int_{G} \chi_{E} \operatorname{div}_{H} \zeta d g=\int_{B\left(e, R_{o}\right)} \chi_{E} f \operatorname{div}_{H} \zeta d g \\
& =\int_{B\left(e, R_{o}\right)} \chi_{E} \operatorname{div}_{H}(f \zeta) d g-\int_{B\left(e, R_{o}\right)} \chi_{E}<\nabla_{H} f, \zeta>d g \\
& =\int_{B\left(e, R_{o}\right)} \chi_{E} \operatorname{div}_{H}(f \zeta) d g \leq P_{H}\left(E, B\left(e, R_{o}\right)\right) .
\end{aligned}
$$

Taking the supremum over all $\zeta \in \mathcal{F}(\boldsymbol{G} ; H \boldsymbol{G})$ we reach the conclusion $P_{H}\left(E, B\left(e, R_{o}\right)\right) \geq$ $P_{H}(E, G)$, thus obtaining (2.3). 
In the next result we extend the isoperimetric inequality from $C^{1}$ to bounded $H$-Caccioppoli sets.

Theorem 2.6. Let $\boldsymbol{G}$ be a Carnot group with homogeneous dimension $Q$. There exists a constant $C_{i s o}(\boldsymbol{G})>0$ such that for every bounded $H$-Caccioppoli set $E \subset \boldsymbol{G}$ one has

$$
|E|^{(Q-1) / Q} \leq C_{i s o}(\boldsymbol{G}) P_{H}(E ; \boldsymbol{G}) \text {. }
$$

Proof. In CDG] it was proved that Theorem 2.2 implies the following Sobolev inequality of Gagliardo-Nirenberg type: for every $u \in C_{0}^{1}\left(B\left(g_{o}, R\right)\right)$

$$
\left\{\int_{B\left(g_{o}, R\right)}|u|^{Q /(Q-1)} d g\right\}^{(Q-1) / Q} \leq C \frac{R}{\left|B\left(g_{o}, R\right)\right|^{1 / Q}} \int_{B\left(g_{o}, R\right)}\left|\nabla_{H} u\right| d g .
$$

If now $u \in B V_{H}\left(B\left(g_{o}, R\right)\right)$, with supp $u \subset B\left(g_{o}, R\right)$, then by Theorem 2.3 there exists a sequence $\left\{u_{k}\right\}_{k \in \mathbb{N}} \in C_{0}^{\infty}\left(B\left(g_{o}, R\right)\right)$ such that $u_{k} \rightarrow u$ in $L^{1}\left(B\left(g_{o}, R\right)\right)$, and $\operatorname{Var}_{H}\left(u_{k} ; B\left(g_{o}, R\right)\right) \rightarrow$ $\operatorname{Var}_{H}\left(u ; B\left(g_{o}, R\right)\right)$, as $k \rightarrow \infty$. Passing to a subsequence, we can assume that $u_{k}(g) \rightarrow u(g)$, for $d g$-a.e. $g \in B\left(g_{o}, R\right)$. Applying (2.4) to $u_{k}$ and passing to the limit we infer from the theorem of Fatou

$$
\left\{\int_{B\left(g_{o}, R\right)}|u|^{Q /(Q-1)} d g\right\}^{(Q-1) / Q} \leq C \frac{R}{\left|B\left(g_{o}, R\right)\right|^{1 / Q}} \operatorname{Var}_{H}\left(u ; B\left(g_{o}, R\right)\right),
$$

for every $u \in B V_{H}\left(B\left(g_{o}, R\right)\right)$, with supp $u \subset B\left(g_{o}, R\right)$. If now $E \subset \bar{E} \subset B\left(g_{o}, R\right)$ is a $H$ Caccioppoli set, then taking $u=\chi_{E}$ in the latter inequality we obtain

$$
|E|^{(Q-1) / Q} \leq C \frac{R}{\left|B\left(g_{o}, R\right)\right|^{1 / Q}} P_{H}\left(E ; B\left(e, R_{o}\right)\right) .
$$

At this point, to reach the desired conclusion we only need to use Lemma 2.5] and observe that $\left|B\left(g_{o}, R\right)\right|=R^{Q}|B(e, 1)|$. We thus obtain the conclusion with $C_{i s o}(\boldsymbol{G})=C|B(e, 1)|^{-1 / Q}$.

The following is a basic consequence of Theorem 2.6 .

Theorem 2.7. Let $\boldsymbol{G}$ be a Carnot group with homogeneous dimension $Q$. With $C_{\text {iso }}(\boldsymbol{G})$ as in Theorem 2.2. one has for any bounded H-Caccioppoli set

$$
|E|^{(Q-1) / Q} \leq C_{i s o}(\boldsymbol{G}) P_{H}(E ; \boldsymbol{G}) .
$$

To establish Theorem [1.3] we next prove that one can remove from Theorem [2.7 without altering the constant $C_{i s o}(\boldsymbol{G})$, the restriction that the $H$-Caccioppoli set be bounded. We recall a useful representation formula. In what follows $N$ indicates the topological dimension of $\boldsymbol{G}$, and $H_{N-1}$ the $(N-1)$-dimensional Hausdorff measure constructed with the Riemannian distance of G.

Lemma 2.8. Let $\Omega \subset \boldsymbol{G}$ be an open set and $E \subset \boldsymbol{G}$ be a $C^{1}$ bounded domain. One has

$$
P_{H}(E ; \Omega)=\int_{\Omega \cap \partial E} \frac{\left|\boldsymbol{N}_{H}\right|}{|\boldsymbol{N}|} d H_{N-1},
$$


where $\boldsymbol{N}_{H}=\sum_{j=1}^{m}<\boldsymbol{N}, X_{j}>X_{j}$ is the projection onto $H \boldsymbol{G}$ of the Riemannian normal $\mathbf{N}$ exterior to $E$. In particular, when

$$
E=\{g \in \boldsymbol{G} \mid \phi(g)<0\}
$$

with $\phi \in C^{1}(\boldsymbol{G})$, and $|\nabla \phi| \geq \alpha>0$ in a neighborhood of $\partial E$, then $\boldsymbol{N}=\nabla \phi$, and therefore $\left|\boldsymbol{N}_{H}\right|=\left|\nabla_{H} \phi\right|$. When $\Omega=\boldsymbol{G}$ we thus obtain in particular

$$
P_{H}(E ; \boldsymbol{G})=\int_{\partial E} \frac{\left|\nabla_{H} \phi\right|}{|\nabla \phi|} d H_{N-1}
$$

For the proof of this lemma we refer the reader to CDG. For a detailed study of the perimeter measure in Lemma 2.8 and (2.6), we refer the reader to DGN1, DGN2] and [CG]. We can finally provide the proof of Theorem 1.3

Theorem 2.9. Let $\boldsymbol{G}$ be a Carnot group with homogeneous dimension $Q$. With the same constant $C_{i s o}(\boldsymbol{G})>0$ as in Theorem 2.7, for every $H$-Caccioppoli set $E \subset \boldsymbol{G}$ one has

$$
|E|^{(Q-1) / Q} \leq C_{i s o}(\boldsymbol{G}) P_{H}(E ; \boldsymbol{G}) \text {. }
$$

Proof. In view of Theorem 2.7 we only need to consider the case of an unbounded $H$-Caccioppoli set $E$. If $P_{H}(E ; \boldsymbol{G})=+\infty$ there is nothing to prove, so we assume that $P_{H}(E ; \boldsymbol{G})<+\infty$ and $|E|<+\infty$. We consider the $C^{\infty} H$-balls $B_{H}(e, R)=\{g \in \boldsymbol{G} \mid \rho(g)<R\}$, generated by the pseudo-distance $\rho=\rho_{e}=\Gamma(\cdot, e)^{1 /(2-Q)} \in C^{\infty}(\boldsymbol{G} \backslash\{e\}) \cap C(\boldsymbol{G})$, where $\Gamma(\cdot, e) \in C^{\infty}(\boldsymbol{G} \backslash\{e\})$ is the fundamental solution with singularity at the identity for the sub-Laplacian $\Delta_{H}=\sum_{j=1}^{m} X_{j}^{2}$. For any $R>0$ we have

$$
P_{H}\left(E \cap B_{H}(e, R) ; \boldsymbol{G}\right) \leq P_{H}\left(E ; B_{H}(e, R)\right)+P_{H}\left(B_{H}(e, R) ; E\right) .
$$

Thanks to the smoothness of $B_{H}(e, R)$ we have from Lemma 2.8

$$
P_{H}\left(B_{H}(e, R) ; E\right)=\int_{\partial B_{H}(e, R) \cap E} \frac{\left|\boldsymbol{N}_{H}\right|}{|\boldsymbol{N}|} d H_{N-1}=\int_{\partial B_{H}(e, R) \cap E} \frac{\left|\nabla_{H} \rho\right|}{|\nabla \rho|} d H_{N-1} .
$$

Recalling that $\Gamma(\cdot, e)$ is homogeneous of degree $2-Q$, see [F1], [F2], and therefore $\rho$ is homogeneous of degree one, we infer that for some constant $C(\boldsymbol{G})>0$,

$$
\left|\nabla_{H} \rho\right| \leq C(\boldsymbol{G}) \text {. }
$$

This gives

$$
P_{H}\left(B_{H}(e, R) ; E\right) \leq C(\boldsymbol{G}) \int_{\partial B_{H}(e, R) \cap E} \frac{d H_{N-1}}{|\nabla \rho|} .
$$

By Federer co-area formula [Fe], we obtain

$$
\infty>|E|=\int_{G} \chi_{E} d g=\int_{0}^{\infty} \int_{\partial B_{H}(e, t) \cap E} \frac{d H_{N-1}}{|\nabla \rho|} d t,
$$

therefore there exists a sequence $R_{k} \nearrow \infty$ such that

$$
\int_{\partial B_{H}\left(e, R_{k}\right) \cap E} \frac{d H_{N-1}}{|\nabla \rho|} \underset{k \rightarrow \infty}{\longrightarrow} 0 .
$$

Using (2.10) in (2.9) we find

$$
\lim _{k \rightarrow \infty} P_{H}\left(B_{H}\left(e, R_{k}\right) ; E\right)=0 .
$$


From (2.7), (2.11), we conclude

$$
\limsup _{k \rightarrow \infty} P_{H}\left(E \cap B_{H}\left(e, R_{k}\right) ; \boldsymbol{G}\right) \leq P_{H}(E ; \boldsymbol{G}) .
$$

We next apply Theorem 2.7 to the bounded $H$-Caccioppoli set $E \cap B_{H}\left(e, R_{k}\right)$ obtaining

$$
\left|E \cap B_{H}\left(e, R_{k}\right)\right|^{(Q-1) / Q} \leq C_{i s o}(\boldsymbol{G}) P_{H}\left(E \cap B_{H}\left(e, R_{k}\right) ; \boldsymbol{G}\right) .
$$

Letting $k \rightarrow \infty$ in the latter inequality, from (2.12), and from the relation

$$
\lim _{k \rightarrow \infty}\left|E \cap B_{H}\left(e, R_{k}\right)\right|^{(Q-1) / Q}=|E|^{(Q-1) / Q},
$$

we conclude that

$$
|E|^{(Q-1) / Q} \leq C_{i s o}(\boldsymbol{G}) P_{H}(E ; \boldsymbol{G}) .
$$

This completes the proof.

We close this section with two basic properties of the $H$-perimeter which clearly play a role also in Theorem 1.2

Proposition 2.10. In a Carnot group $\boldsymbol{G}$ one has for every measurable set $E \subset \boldsymbol{G}$ and every $r>0$

$$
P_{H}(E ; \boldsymbol{G})=r^{Q-1} P_{H}\left(\delta_{1 / r} E ; \boldsymbol{G}\right) \text {. }
$$

Proof. Let $E \subset \boldsymbol{G}$ be a measurable set. If $\zeta \in C_{0}^{1}(\boldsymbol{G}, H \boldsymbol{G})$, then the divergence theorem, and a rescaling, give

$$
\int_{E} \operatorname{div}_{H} \zeta d g=\int_{E} \sum_{j=1}^{m} X_{j} \zeta_{j} d g=r^{Q} \int_{E_{r}} \sum_{j=1}^{m} X_{j} \zeta_{j}\left(\delta_{r} g\right) d g,
$$

where we have let $E_{r}=\delta_{1 / r}(E)=\left\{g \in \boldsymbol{G} \mid \delta_{r} g \in E\right\}$. Since

$$
X_{j}\left(\zeta_{j} \circ \delta_{r}\right)=r\left(X_{j} \zeta_{j} \circ \delta_{r}\right)
$$

we conclude

$$
\int_{E} \sum_{j=1}^{m} X_{j} \zeta_{j} d g=r^{Q-1} \int_{E} \sum_{j=1}^{m} X_{j}\left(\zeta_{j} \circ \delta_{r}\right) d g .
$$

Formula (2.15) implies the conclusion.

Proposition 2.10 asserts that the $H$-perimeter scales appropriately with respect to the nonisotropic group dilations. Since on the other hand one has $\left|\delta_{1 / r} E\right|=r^{-Q}|E|$, we easily obtain the following important scale invariance of the isoperimetric quotient.

Proposition 2.11. For any $H$-Caccioppoli set in a Carnot group $\boldsymbol{G}$ one has

$$
\frac{P_{H}(E ; \boldsymbol{G})}{|E|^{(Q-1) / Q}}=\frac{P_{H}\left(\delta_{1 / r} E ; \boldsymbol{G}\right)}{\left|\delta_{1 / r} E\right|^{(Q-1) / Q}}, \quad r>0 .
$$


Another equally important fact, which is however a trivial consequence of the left-invariance on the vector fields $X_{1}, \ldots, X_{m}$, and of the definition of $H$-perimeter, is the translation invariance of the isoperimetric quotient.

Proposition 2.12. For any $H$-Caccioppoli set in a Carnot group $\boldsymbol{G}$ one has

$$
\frac{P_{H}\left(L_{g_{o}}(E) ; \boldsymbol{G}\right)}{\left|L_{g_{o}}(E)\right|^{(Q-1) / Q}}=\frac{P_{H}(E ; \boldsymbol{G})}{|E|^{(Q-1) / Q}}, \quad g_{o} \in \boldsymbol{G},
$$

where $L_{g_{o}} g=g_{o} g$ is the left-translation on the group.

\section{Partial solution of the isoperimetric problem in $\mathbb{H}^{n}$}

The objective of this section is proving Theorems 1.1 and 1.2. This will be accomplished in several steps. First, we introduce the relevant notions and establish some geometric properties of the $\mathrm{H}$-perimeter that are relevant to the isoperimetric profiles. Next, we collect some results from convex analysis and calculus of variations. Finally, we proceed to proving Theorems 1.1 and 1.2. In what follows we adopt the classical non-parametric point of view, see for instance [MM], according to which a $C^{2}$ hypersurface $\mathcal{S} \subset \boldsymbol{G}$ locally coincides with the zero set of a real function. Thus, for every $g_{0} \in \mathcal{S}$ there exists an open set $\mathcal{O} \subset \boldsymbol{G}$ and a function $\phi \in C^{2}(\mathcal{O})$ such that: (i) $|\nabla \phi(g)| \neq 0$ for every $g \in \mathcal{O}$; (ii) $\mathcal{S} \cap \mathcal{O}=\{g \in \mathcal{O} \mid \phi(g)=0\}$. We will always assume that $\mathcal{S}$ is oriented in such a way that for every $g \in \mathcal{S}$ one has

$$
\boldsymbol{N}(g)=\nabla \phi(g)=X_{1} \phi(g) X_{1}+\ldots+X_{m} \phi(g) X_{m}+\ldots+X_{r, 1} \phi(g) X_{r, 1}+\ldots+X_{r, m_{r}} \phi(g) X_{r, m_{r}} .
$$

To justify the second equality the reader should bear in mind that we have endowed $\boldsymbol{G}$ with a left-invariant Riemannian metric with respect to which $\left\{X_{1}, \ldots, X_{m}, \ldots, X_{r, m_{r}}\right\}$ constitute an orthonormal basis. Given a surface $\mathcal{S} \subset \boldsymbol{G}$, we let

$$
p_{i}=<\boldsymbol{N}, X_{i}>, \quad i=1, \ldots, m,
$$

and define the angle function

$$
W=\sqrt{p_{1}^{2}+\ldots+p_{m}^{2}} .
$$

The motivation for the name comes from the fact that, if $U \angle V$ denotes the angle between two vector fields $U, V$ on $\boldsymbol{G}$, then

$$
\cos \left(\boldsymbol{\nu}_{H} \angle \boldsymbol{N}\right)=\frac{<\boldsymbol{\nu}_{H}, \boldsymbol{N}>}{|\boldsymbol{N}|}=\frac{W}{|\boldsymbol{N}|} .
$$

The characteristic locus of $\mathcal{S}$ is the closed set

$$
\Sigma=\{g \in \mathcal{S} \mid W(g)=0\}=\left\{g \in \mathcal{S} \mid H_{g} \boldsymbol{G} \subset T_{g} \mathcal{S}\right\} .
$$

On the set $\mathcal{S} \backslash \Sigma$ we define the horizontal Gauss map by

$$
\boldsymbol{\nu}_{H}=\bar{p}_{1} X_{1}+\ldots+\bar{p}_{m} X_{m},
$$


where we have let

$$
\bar{p}_{1}=\frac{p_{1}}{W}, \quad \bar{p}_{m}=\frac{p_{m}}{W}, \quad \text { so that } \quad\left|\boldsymbol{\nu}_{H}\right|^{2}=\bar{p}_{1}^{2}+\ldots+\bar{p}_{m}^{2} \equiv 1 \quad \text { on } \quad \mathcal{S} \backslash \Sigma .
$$

Hereafter, when we say that a function $u$ belongs to the class $C^{k}(\mathcal{S})$, we mean that $u \in C(\mathcal{S})$ and that for every $g_{0} \in \mathcal{S}$, there exist an open set $\mathcal{O} \subset \mathbb{H}^{1}$, such that $u$ coincides with the restriction to $\mathcal{S} \cap \mathcal{O}$ of a function in $C^{k}(\mathcal{O})$. The tangential horizontal gradient of a function $u \in C^{1}(\mathcal{S})$ is defined as follows

$$
\delta_{H} u=\nabla_{H} u-<\nabla_{H} u, \nu_{H}>\boldsymbol{\nu}_{H} .
$$

The definition of $\delta_{H} u$ is well-posed since it is noted in DGN4 that $\delta_{H} u$ only depends on the values of $u$ on $\mathcal{S}$. Since $\left|\boldsymbol{\nu}_{H}\right| \equiv 1$ on $\mathcal{S} \backslash \Sigma$, we clearly have $\left\langle\delta_{H} u, \boldsymbol{\nu}_{H}\right\rangle=0$, and therefore

$$
\left|\delta_{H} u\right|^{2}=\left|\nabla_{H} u\right|^{2}-<\nabla_{H} u, \nu_{H}>^{2} .
$$

Given a point $g_{0} \in \mathcal{S} \backslash \Sigma$, the horizontal tangent space of $\mathcal{S}$ at $g_{0}$ is defined by

$$
T_{H, g_{0}}(\mathcal{S})=\left\{\boldsymbol{v} \in H_{g_{0}} \boldsymbol{G} \mid<\boldsymbol{v}, \boldsymbol{\nu}_{H}\left(g_{0}\right)>=0\right\} .
$$

For instance, when $\boldsymbol{G}=\mathbb{H}^{1}$, then a basis for $T_{H, g_{0}}(\mathcal{S})$ is given by the single vector field

$$
\boldsymbol{\nu}_{H}^{\perp}=\bar{p}_{2} X_{1}-\bar{p}_{1} X_{2} .
$$

Given a function $u \in C^{1}(\mathcal{S})$ one clearly has $\delta_{H} u\left(g_{0}\right) \in T_{H, g_{0}}(\mathcal{S})$. We next recall some basic definitions from DGN4.

Definition 3.1. Given a Carnot group $\boldsymbol{G}$, and a $C^{k}$ oriented hypersurface $\mathcal{S} \subset \boldsymbol{G}, k \geq 2$, with characteristic set $\Sigma$, and given a point $g_{0} \in \mathcal{S} \backslash \Sigma$, we define the horizontal second fundamental form $I I_{H}: T_{H, g_{0}} \mathcal{S} \rightarrow T_{H, g_{0}} \mathcal{S}$ of $\mathcal{S}$ at $g_{0}$ as follows. For every $\boldsymbol{u}, \boldsymbol{v} \in T_{H, g_{0}} \mathcal{S}$ we let

$$
\left.\left.I I_{H}(\boldsymbol{u}, \boldsymbol{v})=\frac{1}{2}\left\{<S_{H}(\boldsymbol{u}), \boldsymbol{v}\right\rangle+<S_{H}(\boldsymbol{v}), \boldsymbol{u}\right\rangle\right\},
$$

where $S_{H}: T_{H} \mathcal{S} \rightarrow T_{H} \mathcal{S}$ is the linear operator whose matrix is given by the product of the two $m \times m$ matrices

$$
S_{H}=\frac{1}{W}\left\{\boldsymbol{\nu}_{H} \otimes \boldsymbol{\nu}_{H}-I d\right\}\left[\frac{X_{i} p_{j}+X_{j} p_{i}}{2}\right]_{i, j=1, \ldots, m},
$$

where $m=\operatorname{dim}\left(V_{1}\right)$ is the dimension of the horizontal layer of the Lie algebra of $\boldsymbol{G}$, and $p_{i}=<\boldsymbol{N}, X_{i}>, i=1, \ldots, m$. We call the symmetric operator $\frac{1}{2}\left(S_{H}+S_{H}^{t}\right)$ associated with the quadratic form $I I_{H}$ the horizontal shape operator. We define the horizontal principal curvatures of $\mathcal{S}$ at $g_{0}$ as

$$
\kappa_{i}=I I_{H}\left(\boldsymbol{e}_{i}, \boldsymbol{e}_{i}\right), \quad i=1, \ldots, m-1,
$$

where $\left\{\boldsymbol{e}_{1}, \ldots, \boldsymbol{e}_{m-1}\right\}$ denotes an orthonormal basis of $T_{H, g_{0}} \mathcal{S}$.

Definition 3.2. We define the $H$-mean curvature of $\mathcal{S}$ at a point $g_{0} \in \mathcal{S} \backslash \Sigma$ as follows

$$
\mathcal{H}=-\operatorname{trace} \frac{1}{2}\left(S_{H}+S_{H}^{t}\right)=-\operatorname{trace} S_{H} .
$$

If $g_{0} \in \Sigma$ we let

$$
\mathcal{H}\left(g_{0}\right)=\lim _{g \rightarrow g_{0}, g \in \mathcal{S} \backslash \Sigma} \mathcal{H}(g),
$$


provided that such limit exists, finite or infinite. We do not define the $H$-mean curvature at those points $g_{0} \in \Sigma$ at which the limit does not exist.

We recall that is was proved in $\left[\mathrm{B}\right.$, [Ma that $\mathcal{H}^{Q-1}(\Sigma)=0$, where $\mathcal{H}^{s}$ denotes the $s$ dimensional Hausdorff measure associated with the horizontal or Carnot-Carathéodory distance of $\boldsymbol{G}$, and $Q$ indicates the homogeneous dimension of $\boldsymbol{G}$. We also recall the earlier result of Derridj De1, De2, which states that when $\mathcal{S}$ is $C^{\infty}$ the standard surface measure of $\Sigma$ vanishes.

Definition 3.3. We say that a $C^{2}$ hypersurface $\mathcal{S}$ has constant $H$-mean curvature if $\mathcal{H}$ is globally defined on $\mathcal{S}$, and $\mathcal{H} \equiv$ const. We say that $\mathcal{S}$ is $H$-minimal if $\mathcal{H} \equiv 0$.

Minimal surfaces have been recently studied in $[\mathrm{Pa}], \mathrm{GP}$, [CHMY], $\mathrm{CH}]$. These papers contain also a complete solution of the Bernstein type problem for the Heisenberg group $\mathbb{H}^{1}$. The following result is taken from DGN4].

Proposition 3.4. The $H$-mean curvature coincides with the function

$$
\mathcal{H}=\sum_{i=1}^{m} \delta_{H, i} \bar{p}_{i}=\sum_{i=1}^{m} X_{i} \bar{p}_{i} .
$$

Here, $\bar{p}_{i}=p_{i} / W$, and the second equality is justified by the fact that, since $\sum_{i=1}^{m} \bar{p}_{i}^{2} \equiv 1$, one has

$$
\sum_{i=1}^{m} \delta_{H, i} \bar{p}_{i}=\sum_{i=1}^{m} X_{i} \bar{p}_{i}-\sum_{j=1}^{m} \bar{p}_{j} \sum_{i=1}^{m} X_{j}\left(\frac{\bar{p}_{i}^{2}}{2}\right)=\sum_{i=1}^{m} X_{i} \bar{p}_{i} .
$$

For instance, when $\boldsymbol{G}=\mathbb{H}^{1}$, then according to Proposition 3.4, the $H$-mean curvature of $\mathcal{S}$ is given by

$$
\mathcal{H}=\sum_{i=1}^{2} \delta_{H, i} \nu_{H, i}=\delta_{H, 1}(\bar{p})+\delta_{H, 2}(\bar{q})=X_{1} \bar{p}_{1}+X_{2} \bar{p}_{2}, \quad \text { on } \quad \mathcal{S} \backslash \Sigma .
$$

In this situation, given a $C^{2}$ surface $\mathcal{S} \subset \mathbb{H}^{1}$, there is only one horizontal principal curvature $\kappa_{1}\left(g_{0}\right)$ at every $g_{0} \in \mathcal{S} \backslash \Sigma$. Since in view of (3.8) the vector $\boldsymbol{\nu}_{H}^{\perp}\left(g_{0}\right)$ constitutes an orthonormal basis of $T_{H, g_{0}}(\mathcal{S})$, according to Definition 3.1 we have

$$
\kappa_{1}\left(g_{0}\right)=I I_{H}\left(\boldsymbol{\nu}_{H}^{\perp}\left(g_{0}\right), \boldsymbol{\nu}_{H}^{\perp}\left(g_{0}\right)\right) .
$$

One can verify, see DGN4, that the right-hand side of the latter equation equals $-\mathcal{H}\left(g_{0}\right)$. We recall one more result concerning the $H$-mean curvature that will be useful in the proof of Proposition 3.27. Details can be found in DGN4].

Proposition 3.5. Suppose $\mathcal{S} \subset \mathbb{H}^{n}$ is a level set of a function $\phi$ that takes the form

$$
\phi(z, t)=t-u\left(\frac{|z|^{2}}{4}\right)
$$

for some $C^{2}$ function $u:[0, \infty) \rightarrow \mathbb{R}$. For every point point $g=(z, t) \in \mathcal{S}$ such that $z \neq 0$ the $H$-mean curvature at $g$ is given by

$$
\mathcal{H}=-\frac{2 s u^{\prime \prime}(s)+(Q-3) u^{\prime}(s)\left(1+u^{\prime}(s)^{2}\right)}{2 \sqrt{s}\left(1+u^{\prime}(s)^{2}\right)^{3 / 2}}, \quad s=\frac{|z|^{2}}{4} .
$$


In Proposition 3.5 the hypothesis $z \neq 0$ is justified the fact that, under the given assumptions, if $\mathcal{S}$ intersects the $t$-axis in $\mathbb{H}^{n}$, then the points of intersections are necessarily characteristic for $\mathcal{S}$.

Hereafter in this paper, we restrict our attention to $\boldsymbol{G}=\mathbb{H}^{n}$. In Definition 3.3, following the classical tradition, we have called a hypersurface $H$-minimal if its $H$-mean curvature vanishes identically. However, in the classical setting the measure theoretic definition of minimality is also based on the notion of local minimizer of the area functional. In the paper [DGN4] we have proved that there is a corresponding sub-Riemannian counterpart of such interpretation based on appropriate first and second variation formulas for the $H$-perimeter. For instance, the following first variation formula holds in the Heisenberg group $\mathbb{H}^{1}$.

Theorem 3.6. Let $\mathcal{S} \subset \mathbb{H}^{1}$ be an oriented $C^{2}$ surface, then the first variation of the $H$-perimeter with respect to the deformation

$$
J_{\lambda}(g)=g+\lambda \mathcal{X}(g)=g+\lambda\left(a(g) X_{1}+b(g) X_{2}+k(g) T\right), \quad g=(x, y, t) \in \mathcal{S},
$$

is given by

$$
\left.\frac{d}{d \lambda} P_{H}\left(\mathcal{S}^{\lambda}\right)\right|_{\lambda=0}=\int_{\mathcal{S}} \mathcal{H} \frac{\cos (\mathcal{X} \angle \boldsymbol{N})}{\cos \left(\boldsymbol{\nu}_{H} \angle \boldsymbol{N}\right)}|\mathcal{X}| d \sigma_{H}
$$

where $\angle$ denotes the angle between vectors in the inner product $\langle\cdot, \cdot\rangle$. In particular, $\mathcal{S}$ is stationary with respect to (3.15) if and only if it is H-minimal.

Versions of Theorem 3.6 have also been obtained independently by other people. An approach based on motion by $H$-mean curvature can be found in $[\mathrm{BC}$. When $a=\bar{p} h, b=\bar{q} h$, and $h \in C_{0}^{\infty}(\mathcal{S} \backslash \Sigma)$, then a proof based on CR-geometry can be found in CHMY]. A Riemannian geometry proof, valid in any $\mathbb{H}^{n}$, can be found in $[\mathrm{RR}]$.

In what follows we set

$$
\mathbb{H}_{+}^{n}=\left\{(z, t) \in \mathbb{H}^{n} \mid t>0\right\}, \quad \mathbb{H}_{-}^{n}=\left\{(z, t) \in \mathbb{H}^{n} \mid t<0\right\} .
$$

Consider a domain $\Omega \subset \mathbb{R}^{2 n}$ and a $C^{1}$ function $u: \Omega \rightarrow[0, \infty)$. We assume that $E \subset \mathbb{H}^{n}$ is a $C^{1}$ domain for which

$$
E \cap \mathbb{H}_{+}^{n}=\left\{(z, t) \in \mathbb{H}^{n} \mid z \in \Omega, 0<t<u(z)\right\} .
$$

For $z=(x, y) \in \mathbb{R}^{2 n}$, we set $z^{\perp}=(y,-x)$. Indicating with $\phi(z, t)=t-u(z)$ the defining function of $E \cap \mathbb{H}_{+}^{n}$, a simple computation gives

$$
\begin{aligned}
\left|\nabla_{H} \phi\right|= & =\sqrt{\left|\nabla_{x} u+\frac{y}{2}\right|^{2}+\left|\nabla_{y} u-\frac{x}{2}\right|^{2}} \\
& =\left|\nabla_{z} u+\frac{z^{\perp}}{2}\right| .
\end{aligned}
$$

Invoking the representation formula (2.6) for the $H$-perimeter, which presently gives

$$
P_{H}\left(E ; \mathbb{H}_{+}^{n}\right)=\int_{\partial E \cap \mathbb{H}_{+}^{n}} \frac{\left|\nabla_{H} \phi\right|}{|\nabla \phi|} d H_{2 n},
$$


and keeping in mind that $\nabla \phi=\sqrt{1+\left|\nabla_{z} u\right|^{2}}$, and that $d H_{2 n}=\sqrt{1+\left|\nabla_{z} u\right|^{2}} d z$, we obtain

$$
P_{H}\left(E ; \mathbb{H}_{+}^{n}\right)=\int_{\Omega}\left|\nabla_{z} u+\frac{z^{\perp}}{2}\right| d z=\int_{\Omega} \sqrt{\left|\nabla_{z} u\right|^{2}+\frac{|z|^{2}}{4}+<\nabla_{z} u, z^{\perp}>} d z .
$$

In what follows, we establish some invariance and symmetry properties of the $H$-perimeter. Such properties in turn play a critical role in the proof of Theorem 1.1. Consider the map $\mathcal{O}: \mathbb{H}^{n} \rightarrow \mathbb{H}^{n}$ defined by

$$
\mathcal{O}(x, y, t)=(y, x,-t) .
$$

It is easy to see that $\mathcal{O}$ preserves Lebesgue measure (which is a bi-invariant Haar measure on $\mathbb{H}^{n}$ ). In fact, the map $\mathcal{O}$ is a group and Lie algebra automorphism of $\mathbb{H}^{n}$. Such map is called inversion in [F3], p.20.

Lemma 3.7. Let $E \subset \mathbb{H}^{n}$ be measurable, then $|E|=|\mathcal{O}(E)|$.

Proof. Observe that $\left|\operatorname{det}\left(J_{a c_{\mathcal{O}}}\right)\right|=1$, and therefore

$$
|\mathcal{O}(E)|=\int_{\mathcal{O}(E)} d g=\int_{E}\left|\operatorname{det}\left(J a c_{\mathcal{O}}\right)\right| d g=|E|
$$

Perhaps, it is somewhat surprising that, under no additional symmetry assumptions, $\mathcal{O}$ also preserves the $H$-perimeter (note that the reflection alone $(x, y, t) \rightarrow(x, y,-t)$ does not preserve the $H$-perimeter!).

Lemma 3.8. Let $E \subset \mathbb{H}^{n}$ be a piecewise $C^{1}$ domain with $P_{H}\left(E, \mathbb{H}^{n}\right)<\infty$, then

$$
P_{H}\left(\mathcal{O}(E), \mathbb{H}^{n}\right)=P_{H}\left(E, \mathbb{H}^{n}\right) \text {. }
$$

Proof. We give the proof for the first Heisenberg group $\mathbb{H}^{1}$, leaving it to the reader to provide the easy modifications necessary to cover the higher-dimensional case. Suppose that $E$ is as in (2.5) for some piecewise $C^{1}$ function $\phi: \mathbb{H}^{1} \rightarrow \mathbb{R}$ with $|\nabla \phi| \geq \alpha>0$ in a neighborhood of $\partial E$. Let $W \subset \mathbb{R}^{2}$ be a connected open set, and $A: W \rightarrow A(W) \subset \partial E$ be a coordinate patch of a smooth part of $\partial E$, then

$$
\int_{A(W)} \frac{\left|\nabla_{H} \phi\right|}{|\nabla \phi|} d \sigma=\int_{W} \frac{\left|\nabla_{H} \phi \circ A\right|}{|\nabla \phi \circ A|} \sqrt{E_{A} G_{A}-F_{A}^{2}} d \alpha d \beta
$$

where $d \sigma=\sqrt{E_{A} G_{A}-F_{A}^{2}} d \alpha d \beta$ is the standard Riemannian volume density induced by $A$ on $A(W)$. On the other hand, it is easy to see that the defining function $\psi$ of $\mathcal{O}(E)$ is given by $\psi=\phi \circ \mathcal{O}^{-1}$ and $\tilde{A} \stackrel{\text { def }}{=} \mathcal{O} \circ A: W \rightarrow \partial \mathcal{O}(E)$ is a coordinate patch on a smooth portion of $\partial \mathcal{O}(E)$. We thus have

$$
\int_{\tilde{A}(W)} \frac{\left|\nabla_{H} \psi\right|}{|\nabla \psi|} d \sigma=\int_{W} \frac{\left|\nabla_{H}\left(\phi \circ \mathcal{O}^{-1}\right) \circ \tilde{A}\right|}{\left|\nabla\left(\phi \circ \mathcal{O}^{-1}\right) \circ \tilde{A}\right|} \sqrt{E_{\tilde{A}} G_{\tilde{A}}-F_{\tilde{A}}^{2}} \quad d \alpha d \beta,
$$

where $\sqrt{E_{\tilde{A}} G_{\tilde{A}}-F_{\tilde{A}}^{2}}$ is the Riemannian volume density induced by $\tilde{A}$ on $\tilde{A}(W)$. From the definition of the map $\mathcal{O}$ it is easy to verify that

(i) $\mathcal{O}^{-1}=\mathcal{O}$. 
(ii) $X_{1} \psi \circ \mathcal{O}=X_{2} \phi, X_{2} \psi \circ \mathcal{O}=X_{1} \phi$. Hence, $\left|\nabla_{H} \psi \circ \tilde{A}\right|=\left|\nabla_{H} \phi \circ A\right|$.

(iii) $\nabla \psi \circ \mathcal{O}=\left(\phi_{y}, \phi_{x},-\phi_{t}\right)$. Hence, $|\nabla \psi \circ \tilde{A}|=|\nabla \phi \circ A|$.

(iv) $E_{A}=E_{\tilde{A}}, G_{A}=G_{\tilde{A}}, F_{A}=F_{\tilde{A}}$.

Using (i)-(iv) we readily conclude that

$$
\int_{A(W)} \frac{\left|\nabla_{H} \phi\right|}{|\nabla \phi|} d \sigma=\int_{\tilde{A}(W)} \frac{\left|\nabla_{H} \psi\right|}{|\nabla \psi|} d \sigma .
$$

Since $\partial E$ is a piecewise $C^{1}$ manifold, both $\partial E$ and $\partial \mathcal{O}(E)$ can be covered by the same sequence of domains of integration $W_{1}, W_{2}, \ldots$ (finite sequence if $\partial E$ is compact, see, e.g., [STh]), and therefore we conclude from (3.19) that

$$
P_{H}\left(E ; \mathbb{H}^{1}\right)=\int_{\partial E} \frac{\left|\nabla_{H} \phi\right|}{|\nabla \phi|} d \sigma=\int_{\partial \mathcal{O}(E)} \frac{\left|\nabla_{H} \psi\right|}{|\nabla \psi|} d \sigma=P_{H}\left(\mathcal{O}(E) ; \mathbb{H}^{1}\right)
$$

Remark 3.9. The proof of Lemma 3.8 shows in fact that for any open set $\Omega \subset \mathbb{H}^{n}$ one has $P_{H}(\mathcal{O}(E) ; \mathcal{O}(\Omega))=P_{H}(E ; \Omega)$.

Using the above two lemmas, we can establish the following symmetry result for isoperimetric sets whose intersection with the hyperplane $\{t=0\}$ is a $(2 n)$-dimensional ball.

Theorem 3.10. Let $E \subset \mathbb{H}^{n}$ be a bounded open set such that $\partial E \cap \mathbb{H}_{+}^{n}$ and $\partial E \cap \mathbb{H}_{-}^{n}$ are $C^{1}$ hypersurfaces, and assume that $E$ satisfies the following condition: there exists $R>0$ such that

$$
E \cap\{t=0\}=B(0, R) .
$$

Suppose $E$ is an isoperimetric set satisfying $\left|E \cap \mathbb{H}_{+}^{n}\right|=\left|E \cap \mathbb{H}_{-}^{n}\right|=|E| / 2$, then

$$
P_{H}\left(E ; \mathbb{H}_{+}^{n}\right)=P_{H}\left(E ; \mathbb{H}_{-}^{n}\right) .
$$

Proof. We argue by contradiction, and assume that $E$ satisfies $\left|E \cap \mathbb{H}_{+}^{n}\right|=\left|E \cap \mathbb{H}_{-}^{n}\right|=|E| / 2$, but

$$
P_{H}\left(E ; \mathbb{H}_{+}^{n}\right)<P_{H}\left(E ; \mathbb{H}_{-}^{n}\right) .
$$

Let $\mathcal{O}$ be the mapping in Lemma 3.8 and

$$
F=\left(E \cap \mathbb{H}_{+}^{n}\right) \cup \mathcal{O}\left(E \cap \mathbb{H}_{+}^{n}\right) .
$$

That is, $F$ is obtained from $E$ by taking the top half part of $E$ together with the "twisted reflection" of the top part of $E$ across the horizontal plane $\left\{(z, t) \in \mathbb{H}^{n} \mid t=0\right\}$. Let $\phi \in C^{1}\left(\mathbb{H}^{n}\right)$ be a defining function for $E$. We define $\psi: \mathbb{H}^{n} \rightarrow \mathbb{R}$ by

$$
\psi(x, y, t)= \begin{cases}\phi(x, y, t) & \text { if } t \geq 0 \\ \phi \circ \mathcal{O}(x, y, t) & \text { if } t<0 .\end{cases}
$$

Clearly, $\psi$ is a piecewise $C^{1}$ function, and $F=\left\{g \in \mathbb{H}^{n} \mid \psi(g)<0\right\}$. Next, observing that the map $\mathcal{O}$ preserves $|z|$, we conclude

$$
\mathcal{O}(E \cap\{t=0\})=\mathcal{O}(B(0, R))=E \cap\{t=0\} .
$$


Hence, the construction of the set $F$ does not introduce any additional $H$-perimeter. Since $\partial F$ is piecewise $C^{1}$, we have by Lemma 3.8, Remark 3.9 and by (3.21)

$$
\begin{aligned}
P_{H}\left(F ; \mathbb{H}^{n}\right) & =P_{H}\left(F ; \mathbb{H}_{+}^{n}\right)+P_{H}\left(F ; \mathbb{H}_{-}^{n}\right)=P_{H}\left(E ; \mathbb{H}_{+}^{n}\right)+P_{H}\left(\mathcal{O}\left(E \cap \mathbb{H}_{+}^{n}\right) ; \mathbb{H}_{-}^{n}\right) \\
& =P_{H}\left(E ; \mathbb{H}_{+}^{n}\right)+P_{H}\left(\mathcal{O}\left(E \cap \mathbb{H}_{+}^{n}\right) ; \mathcal{O}\left(\mathbb{H}_{+}^{n}\right)\right) \\
& =2 P_{H}\left(E ; \mathbb{H}_{+}^{n}\right) \\
& <P_{H}\left(E ; \mathbb{H}^{n}\right) .
\end{aligned}
$$

On the other hand, by the assumption on $|E|$ and Lemma 3.7. we have $|F|=\left|E \cap \mathbb{H}_{+}^{n}\right|+\left|\mathcal{O}\left(E \cap \mathbb{H}_{+}^{n}\right)\right|=\left|E \cap \mathbb{H}_{+}^{n}\right|+\left|E \cap \mathbb{H}_{+}^{n}\right|=\left|E \cap \mathbb{H}_{+}^{n}\right|+\left|E \cap \mathbb{H}_{-}^{n}\right|=|E|$.

This proves that $E$ is not an isoperimetric set, thus giving a contradiction.

We now introduce the relevant functional class for our problem. The space of competing functions $\mathcal{D}$ is defined as follows.

Definition 3.11. We let

$$
\begin{array}{r}
\mathcal{D}=\left\{u: \mathbb{R}^{2 n} \rightarrow \mathbb{R} \mid \text { there exists } R>0 \text { such that } \operatorname{supp}(u)=\bar{B}(0, R),\right. \\
\left.u \geq 0 \text { in } B(0, R), u \in C^{1}(B(0, R)) \cap C(\bar{B}(0, R))\right\} .
\end{array}
$$

We remark that, although $\mathcal{D}$ is not a linear space (due to the condition $u \geq 0$ ), if $u, v \in \mathcal{D}$ and $\epsilon \geq 0$, then $u+v \in \mathcal{D}$ and $\epsilon u \in \mathcal{D}$. Following classical ideas from Calculus of Variation, we next introduce the admissible variations for the problem at hand, see $\mathrm{GH}]$ and $\mathrm{Tr}$.

Definition 3.12. Given $\mathcal{D}$, the space of competing functions, we define

$$
\begin{aligned}
\mathcal{A}_{\mathcal{D}}=\left\{\phi: \mathbb{R}^{2 n} \rightarrow \mathbb{R} \mid\right. & \phi \text { has compact support, } \\
& \left.\phi \in C^{1}(\operatorname{supp}(\phi)) \cap C(\overline{\operatorname{supp}(\phi)}) \text { and for all } u \in \mathcal{D}, u+\phi \in \mathcal{D}\right\} .
\end{aligned}
$$

The set $\mathcal{A}_{\mathcal{D}}$ is called a space of admissible variations of $\mathcal{D}$.

The following properties of $\mathcal{A}_{\mathcal{D}}$ are obvious. First, $\mathcal{A}_{\mathcal{D}} \neq \varnothing$ since if $u \in \mathcal{D}$ we see that if $\phi(z)=u(\eta z)$ for some $\eta>0$, then $\phi \in \mathcal{A}_{\mathcal{D}}$. Also, if $\phi \in \mathcal{A}_{\mathcal{D}}, 0 \leq \epsilon \leq 1$, then $\epsilon \phi \in \mathcal{A}_{\mathcal{D}}$.

Remark 3.13. The set $\mathcal{A}_{\mathcal{D}}$ can be defined in a more general setting. If $\mathcal{D}$ is a subset of a linear space $\mathcal{V}$, then

$$
\mathcal{A}_{\mathcal{D}}=\{\phi \in \mathcal{V} \mid \text { for all } u \in \mathcal{D}, u+\phi \in \mathcal{D}\}
$$

Now, for $u \in \mathcal{D}$ we let

$$
G[u]=\int_{\operatorname{supp}(u)} u(z) d z=\int_{B(0, R)} u(z) d z .
$$

With (3.18) in mind, we define for such $u$

$$
J[u]=\int_{\text {supp }(u)} \sqrt{\left|\nabla_{z} u\right|^{2}+\frac{|z|^{2}}{4}+<\nabla_{z} u, z^{\perp}>} .
$$


In the class of $C^{1}$ graphs over $\mathbb{R}^{2 n} \times\{0\}$, the isoperimetric problem consists in minimizing the functional $J[u]$ subject to the constraint that $G[u]=V$, where $V>0$ is given and $B(0, R)$ is replaced by a domain $\Omega$. We emphasize that finding the section of the isoperimetric profile with the hyperplane $\{t=0\}$, i.e., finding the domain the radius $R>0$ of the ball $B(0, R)$, or more in general the domain $\Omega$, constitutes here part of the problem. For a general domain $\Omega$, this seems a difficult question at the moment. To make further progress we restrict the class of domains $E$ by imposing that their section with the hyperplane $\{t=0\}$ be a ball, i.e., we assume that, given $E \in \mathcal{E}$, there exists $R=R(E)>0$ such that $\Omega=B(0, R)$. Under this hypothesis, we can appeal to Theorem 3.10. The latter implies that it suffices to solve the following variational problem: given $V>0$, find $R_{o}>0$ and $u_{o} \in \mathcal{D}$ with $\operatorname{supp}\left(u_{o}\right)=B\left(0, R_{o}\right)$ for which the following holds

$$
J\left[u_{o}\right]=\min \{J[u] \mid u \in \mathcal{D}\} \quad \text { and } \quad G\left[u_{o}\right]=\frac{V}{2} .
$$

To reduce the problem (3.25) to one without constraint, we will apply the following standard version of Lagrange multiplier theorem (see, e.g., Proposition 2.3 in $[\mathrm{Tr}$ ).

Proposition 3.14. Let $\mathcal{D}$ be a subset of a normed vector space $\mathcal{V}$, and consider functionals $\mathcal{F}$, $\mathcal{G}_{1}, \mathcal{G}_{2}, \ldots, \mathcal{G}_{k}$ defined on $\mathcal{D}$. Suppose there exist constants $\lambda_{1}, \ldots, \lambda_{k} \in \mathbb{R}$, and $u_{o} \in \mathcal{D}$, such that $u_{o}$ minimizes (uniquely)

$$
\mathcal{F}+\lambda_{1} \mathcal{G}_{1}+\lambda_{2} \mathcal{G}_{2}+\cdots+\lambda_{k} \mathcal{G}_{k}
$$

on $\mathcal{D}$, then $u_{o}$ minimizes $\mathcal{F}$ (uniquely) when restricted to the set

$$
\left\{u \in \mathcal{D} \mid \mathcal{G}_{j}[u]=\mathcal{G}_{j}\left[u_{o}\right], j=1, \ldots, k\right\}
$$

Remark 3.15. The procedure of applying the above proposition to solving a problem of the type

$$
\left\{\begin{array}{l}
\text { minimize }\{\mathcal{F}[u] \mid u \in \mathcal{D}\}, \\
\text { subject to the constraints }
\end{array} \mathcal{G}_{1}[u]=V_{1}, \ldots, \mathcal{G}_{k}[u]=V_{k},\right.
$$

consists of two main steps. First, one needs to show that constants $\lambda_{1}, \ldots, \lambda_{k}$ and $a u_{o} \in \mathcal{D}$ can be found in such a way that $u_{o}$ solves the Euler-Lagrange equation of (3.26), and $u_{o}$ satisfies $\mathcal{G}_{1}\left[u_{o}\right]=V_{1}, \ldots, \mathcal{G}_{k}\left[u_{o}\right]=V_{k}$. Finally, one proves that the solution $u_{o}$ of the Euler-Lagrange equation is indeed a minimizer of (3.26). If the functional involved possesses appropriate convexity properties, then one can show that such minimizer $u_{o}$ is unique.

We then proceed with the first step outlined in the Remark 3.15. In what follows, with $z \in \mathbb{R}^{2 n}, u \in \mathbb{R}$, and $p=\left(p_{1}, p_{2}\right) \in \mathbb{R}^{2 n}$, we let

$$
\left\{\begin{array}{l}
f(z, u, p)=f(z, p)=\sqrt{\left|p_{1}+\frac{y}{2}\right|^{2}+\left|p_{2}-\frac{x}{2}\right|^{2}}=\left|p+\frac{z^{\perp}}{2}\right| \\
g(z, u, p)=g(u)=u \\
h(z, u, p)=f(z, p)+\lambda g(u) .
\end{array}\right.
$$


The constrained variational problem (3.25) is then equivalent to the following one without constraint (provided the parameter $\lambda$ is appropriately chosen): to minimize the functional

$$
\mathcal{J}[u]=\int_{\text {supp }(u)} h\left(z, u(z), \nabla_{z} u(z)\right) d z=\int_{\text {supp }(u)}\left\{\left|\nabla_{z} u(z)+\frac{z^{\perp}}{2}\right|+\lambda u(z)\right\} d z,
$$

over the set $\mathcal{D}$ defined in (3.22). We easily recognize that the Euler-Lagrange equation of (3.28) is

$$
\operatorname{div}_{z}\left[\frac{\nabla_{z} u+\frac{z^{\perp}}{2}}{\sqrt{\left|\nabla_{z} u\right|^{2}+\frac{|z|^{2}}{4}+<\nabla_{z} u, z^{\perp}>}}\right]=\lambda .
$$

Remark 3.16. Before proceeding we note explicitly that, if we knew a priori that $u \in C^{2}(\Omega)$, considering the $C^{2}$ hypersurface $\mathcal{S}=\left\{(z, t) \in \mathbb{H}^{n} \mid z \in \Omega, t=u(z)\right\}$, and indicating with $\Sigma$ its characteristic set, then $g=(z, t) \notin \Sigma$ if and only if $\left|\nabla_{z} u\right|^{2}+\frac{|z|^{2}}{4}+<\nabla_{z} u, z^{\perp}>\neq 0$. In this situation, it can be recognized that, at every $g \notin \Sigma$, the quantity in the left-hand side of (3.29) represents the $H$-mean curvature $\mathcal{H}$ of $\mathcal{S}$.

As we have said, solving (3.29) on an arbitrary domain of $\Omega \subset \mathbb{R}^{2 n}$ is a difficult task. However, when $\Omega$ is a ball in $\mathbb{R}^{2 n}$, the equation (3.29) admits a remarkable family of spherically symmetric solutions.

Theorem 3.17. Let $\Omega=B(0, R)=\left\{z \in \mathbb{R}^{2 n}|| z \mid<R\right\}$. Given $R>0$, for every

$$
-\frac{Q-2}{R} \leq \lambda<0
$$

the equation (3.29), with the Dirichlet condition $u=0$ on $\partial \Omega$, admits the spherically symmetric solution $u_{R, \lambda} \in \mathcal{D}$, where

$$
u_{R, \lambda}(z)=C_{R, \lambda}+\frac{|z|}{4 \lambda} \sqrt{(Q-2)^{2}-(\lambda|z|)^{2}}-\frac{(Q-2)^{2}}{4 \lambda^{2}} \sin ^{-1}\left(\frac{\lambda|z|}{Q-2}\right),
$$

and

$$
C_{R, \lambda}=-\frac{R}{4 \lambda} \sqrt{(Q-2)^{2}-(\lambda R)^{2}}-\frac{(Q-2)^{2}}{4 \lambda^{2}} \sin ^{-1}\left(\frac{\lambda R}{Q-2}\right)
$$

Proof. We look for a spherically symmetric solution in the form $u(z)=\bar{u}\left(|z|^{2} / 4\right)$, for some function $\bar{u} \in C^{1}\left(\left[0, R^{2} / 4\right)\right) \cap C\left(\left[0, R^{2} / 4\right]\right)$, with $\bar{u}\left(R^{2} / 4\right)=0$. The equation (3.29) becomes

$$
\operatorname{div}_{z}\left[\frac{\bar{u}^{\prime}\left(|z|^{2} / 4\right) z+z^{\perp}}{|z| \sqrt{1+\bar{u}^{\prime}\left(|z|^{2} / 4\right)}}\right]=\lambda \text {. }
$$

Since

$$
\operatorname{div}_{z}\left[\frac{z^{\perp}}{|z| \sqrt{1+\bar{u}^{\prime}\left(|z|^{2} / 4\right)}}\right]=0,
$$

we obtain that (3.33) reduces to the equation

$$
\operatorname{div}_{z}\left[\frac{\bar{u}^{\prime}\left(|z|^{2} / 4\right) z}{|z| \sqrt{1+\bar{u}^{\prime}\left(|z|^{2} / 4\right)}}\right]=\lambda .
$$


The transformation

$$
F(r) \stackrel{\text { def }}{=} \frac{\bar{u}^{\prime}\left(\frac{r^{2}}{4}\right)}{r \sqrt{1+\left(\bar{u}^{\prime}\left(\frac{r^{2}}{4}\right)\right)^{2}}},
$$

turns the nonlinear equation (3.34) into the following linear one

$$
F^{\prime}(r)+\frac{2 n}{r} F(r)=\frac{\lambda}{r},
$$

which is equivalent to

$$
\left(r^{2 n} F\right)^{\prime}=\lambda r^{2 n-1} .
$$

Using the $C^{1}$ smoothness of $u$ at $z=0$ (which implies $\lim _{r \rightarrow 0} r^{2 n} F(r)=0$ ), we can easily integrate the latter equation, obtaining $F(r) \equiv \lambda / 2 n$. Setting $s=r^{2} / 4$ in the latter identity one obtains from (3.35)

$$
\frac{\bar{u}^{\prime}(s)}{\sqrt{1+\left(\bar{u}^{\prime}(s)\right)^{2}}}=\frac{\lambda}{n} \sqrt{s}=\frac{2 \lambda}{Q-2} \sqrt{s} .
$$

Excluding the case of $H$-minimal surfaces (corresponding to $\lambda=0$ ), equation (3.36) gives

$$
\frac{\left(\bar{u}^{\prime}\right)^{2}}{1+\left(\bar{u}^{\prime}\right)^{2}}=\alpha^{2} s
$$

with

$$
\alpha=\frac{2 \lambda}{Q-2}
$$

This in turn implies

$$
\bar{u}^{\prime}(s)= \pm \sqrt{\frac{s}{\beta^{2}-s}}, \quad \text { where } \quad \beta=\frac{1}{\alpha} .
$$

At this point, an observation must be made. We cannot choose the sign in (3.39) arbitrarily. In fact, equation (3.36) implies that $\bar{u}^{\prime}$ does not change sign on the interval $\left[0, R^{2} / 4\right]$, and one has $\bar{u}^{\prime}>0$, or $\bar{u}^{\prime}<0$, according to whether $\alpha>0$ or $\alpha<0$. On the other hand, if the ' + ' branch of the square root were chosen in (3.39), then $\bar{u}$ would be increasing and, since $\bar{u} \geq 0$ on $\left(0, R^{2} / 4\right)$, it would be thus impossible to fulfill the boundary condition $\bar{u}\left(R^{2} / 4\right)=0$.

These considerations show that it must be $\bar{u}^{\prime}<0$ on $\left(0, R^{2} / 4\right)$. We then have to take $\alpha<0$ (hence $\beta<0$ as well), and therefore $\lambda<0$. Equation (3.39) thus becomes

$$
\bar{u}^{\prime}(s)=-\sqrt{\frac{s}{\beta^{2}-s}}, \quad 0 \leq s<\frac{R^{2}}{4} .
$$

We stress that, thanks to the assumption (3.30), and to (3.38), we have that if

$$
0 \leq s<\frac{R^{2}}{4}=\frac{(Q-2)^{2}}{4 \lambda^{2}}=\frac{1}{\alpha^{2}}=\beta^{2},
$$

then the function $\bar{u}^{\prime}$ given by (3.40) is smooth on the interval $\left[0, R^{2} / 4\right)$, and satisfies

$$
\lim _{s \rightarrow\left(\frac{R^{2}}{4}\right)^{-}} \bar{u}^{\prime}(s)=-\infty .
$$


Integrating (3.40) by standard calculus techniques we find for $s \in\left[0, R^{2} / 4\right]$

$$
\begin{aligned}
\bar{u}(s) & =\sqrt{s\left(\beta^{2}-s\right)}-\beta^{2} \tan ^{-1}\left(\sqrt{\frac{s}{\beta^{2}-s}}\right)+C \\
& =C+\sqrt{s\left(\beta^{2}-s\right)}+\beta^{2} \sin ^{-1}\left(\frac{\sqrt{s}}{\beta}\right) .
\end{aligned}
$$

Recalling that $\alpha=\beta^{-1}$, and the equation (3.38), if we impose the condition $\bar{u}\left(R^{2} / 4\right)=0$, we obtain the solution

$$
\bar{u}(s)=C_{R, \lambda}+\frac{\sqrt{s}}{2 \lambda} \sqrt{(Q-2)^{2}-4 \lambda^{2} s}+\frac{(Q-2)^{2}}{4 \lambda^{2}} \sin ^{-1}\left(\frac{2 \lambda \sqrt{s}}{Q-2}\right),
$$

where $C_{R, \lambda}$ is given by (3.32). Setting $u_{R, \lambda}(z)=\bar{u}\left(|z|^{2} / 4\right)$, we finally obtain (3.31) from (3.42). We are finally left with proving that such a $u_{R, \lambda}$ belongs to the class $\mathcal{D}$. This is equivalent to proving that the function $s \rightarrow \bar{u}\left(s^{2} / 4\right)$ is of class $C^{1}$ in the open interval $(-R, R)$. From (3.42) it is clear that we only need to check the continuity of the first derivative of such function at $s=0$. Since the function is even this amounts to proving that $\bar{u}^{\prime}(s) \rightarrow 0$ as $s \rightarrow 0$. But this is obvious in view of (3.40).

From Theorem 3.17 we immediately obtain the following important consequence.

Theorem 3.18. Let $V>0$ be given, and define $R=R(V)>0$ by the formula

$$
R=\left(\frac{(Q-2) \Gamma\left(\frac{Q+2}{2}\right) \Gamma\left(\frac{Q-1}{2}\right)}{\pi^{\frac{Q-1}{2}} \Gamma\left(\frac{Q+1}{2}\right)}\right)^{1 / Q} V^{1 / Q} .
$$

With such choice of $R$, let $\Omega=B(0, R)=\left\{z \in \mathbb{R}^{2 n}|| z \mid<R\right\}$. If we take

$$
\lambda=-\frac{Q-2}{R},
$$

then the equation (3.29), with the Dirichlet condition $u=0$ on $\partial \Omega$, admits the spherically symmetric solution $u_{R} \in \mathcal{D}$, where

$$
u_{R}(z)=\frac{\pi R^{2}}{8}+\frac{|z|}{4} \sqrt{R^{2}-|z|^{2}}-\frac{R^{2}}{4} \sin ^{-1}\left(\frac{|z|}{R}\right) .
$$

Furthermore, such $u_{R}$ satisfies the condition

$$
\int_{\Omega} u_{R}(z) d z=\frac{V}{2}
$$

Proof. The first part of the theorem, up to formula (3.45), is a direct consequence of Theorem 3.17. We only need to prove (3.46). In this respect, keeping in mind the definition (3.43), it will suffice to prove that

$$
\int_{\Omega} u_{R}(z) d z=\frac{\pi^{\frac{Q-1}{2}} \Gamma\left(\frac{Q+1}{2}\right)}{2(Q-2) \Gamma\left(\frac{Q+2}{2}\right) \Gamma\left(\frac{Q-1}{2}\right)} R^{Q} .
$$

To establish (3.47) we note explicitly that $u_{R}(z)=\bar{u}\left(|z|^{2} / 4\right)$, where

$$
\bar{u}(s)=\frac{\pi R^{2}}{8}+\frac{1}{2} \sqrt{s\left(R^{2}-4 s\right)}-\frac{R^{2}}{4} \sin ^{-1}\left(\frac{2 \sqrt{s}}{R}\right) .
$$


One has therefore

$$
\begin{aligned}
\int_{\Omega} u_{R}(z) d z & =\int_{|z|<R} \bar{u}\left(|z|^{2} / 4\right) d z=\sigma_{2 n-1} \int_{0}^{R} \bar{u}\left(r^{2} / 4\right) r^{2 n} \frac{d r}{r} \\
& =2^{2 n-1} \sigma_{2 n-1} \int_{0}^{\frac{R^{2}}{4}} \bar{u}(s) s^{(Q-4) / 2} d s .
\end{aligned}
$$

Integrating by parts the last integral, and using the fact that $\bar{u}\left(R^{2} / 4\right)=0$, that $\bar{u}$ is smooth at 0 , and (3.40) (in which now $\beta^{2}=\frac{R^{2}}{4}$ ), we obtain

$$
\begin{aligned}
\int_{\Omega} u_{R}(z) d z & =\frac{2^{2 n} \sigma_{2 n-1}}{Q-2} \int_{0}^{\frac{R^{2}}{4}} \frac{s^{\frac{Q-1}{2}}}{\sqrt{\frac{R^{2}}{4}-s}} d s \\
& =\frac{2^{2 n+1} \sigma_{2 n-1}}{Q-2} \int_{0}^{\frac{R^{2}}{4}} s^{\frac{Q-2}{2}} \sqrt{\frac{s}{R^{2}-4 s}} d s .
\end{aligned}
$$

With the substitution

$$
t^{2}=\frac{R^{2}-4 s}{s}, \quad d s=\frac{-2 R^{2} t}{\left(4+t^{2}\right)^{2}} d t
$$

the integral (3.49) becomes

$$
\begin{aligned}
\int_{\Omega} u_{R}(z) d z & =\frac{2^{Q} \sigma_{2 n-1} R^{Q}}{Q-2} \int_{0}^{\infty} \frac{1}{\left(4+t^{2}\right)^{\frac{Q+2}{2}}} d t \\
& =\frac{\sigma_{2 n-1} R^{Q}}{4(Q-2)} \int_{\mathbb{R}} \frac{1}{\left(1+t^{2}\right)^{\frac{Q+2}{2}}} d t
\end{aligned}
$$

Now we use the formula

$$
\int_{\mathbb{R}} \frac{d t}{\left(1+t^{2}\right)^{a}}=\pi^{\frac{1}{2}} \frac{\Gamma\left(a-\frac{1}{2}\right)}{\Gamma(a)},
$$

valid for any $a>1 / 2$. We thus obtain

$$
\int_{\Omega} u_{R}(z) d z=\frac{\sigma_{2 n-1} \pi^{\frac{1}{2}} \Gamma\left(\frac{Q+1}{2}\right)}{4(Q-2) \Gamma\left(\frac{Q+2}{2}\right)} R^{Q}
$$

where $\sigma_{2 n-1}$ is the measure of the unit sphere $\mathbb{S}^{n-1}$ in $\mathbb{R}^{2 n}$. Finally, using in the latter equality the fact that

$$
\sigma_{2 n-1}=\frac{2 \pi^{n}}{\Gamma(n)}=\frac{2 \pi^{\frac{Q-2}{2}}}{\Gamma\left(\frac{Q-2}{2}\right)},
$$

we obtain (3.47).

With the problem (3.25) in mind, it is convenient to rephrase part of the conclusion of Theorem 3.18 in the following way. 
Corollary 3.19. Let $V>0$ be given, and for any $R>0$ consider the function $u_{R}$ defined by (3.45). There exists $R=R(V)>0$ (the choice of $R$ is determined by (3.43)) such that with $\Omega=B(0, R)$ one has with $u_{o}=u_{R}$

$$
G\left[u_{o}\right]=\int_{\Omega} u_{o}(z) d z=\frac{V}{2} .
$$

Although the following lemma will not be used until we come to the proof of Theorem [1.2, it is nonetheless appropriate to present it at this moment, since it complements Corollary 3.19,

Lemma 3.20. Let $u_{o}(z)$ be given by (3.45), and $\Omega=\operatorname{supp}\left(u_{o}\right)=B(0, R)$, then

$$
J\left[u_{o}\right]=\int_{\Omega} \sqrt{\left|\nabla_{z} u_{o}\right|^{2}+\frac{|z|^{2}}{4}+<\nabla_{z} u_{o}, z^{\perp}>} d z=\frac{\pi^{\frac{Q-1}{2}} \Gamma\left(\frac{Q-1}{2}\right)}{2 \Gamma\left(\frac{Q}{2}\right) \Gamma\left(\frac{Q-1}{2}\right)} R^{Q-1} .
$$

Proof. We recall that $u_{o}(z)=\bar{u}\left(|z|^{2} / 4\right)$ where $\bar{u}$ is given by (3.48). One has

$$
\nabla_{z} u_{o}(z)=\frac{1}{2} \bar{u}^{\prime}\left(|z|^{2} / 4\right) z
$$

and therefore

$$
\left|\nabla_{z} u_{o}(z)\right|^{2}+\frac{|z|^{2}}{4}+<\nabla_{z} u_{o}(z), z^{\perp}>=\frac{|z|^{2}}{4}\left(1+\bar{u}^{\prime}\left(\frac{|z|^{2}}{4}\right)^{2}\right) .
$$

We thus obtain

$$
\begin{aligned}
& \int_{\Omega} \sqrt{\left|\nabla_{z} u_{o}\right|^{2}+\frac{|z|^{2}}{4}+<\nabla_{z} u_{o}, z^{\perp}>} d z=\frac{1}{2} \int_{|z|<R}|z| \sqrt{1+\bar{u}^{\prime}\left(|z|^{2} / 4\right)^{2}} d z \\
& =\frac{\sigma_{2 n-1}}{2} \int_{0}^{R} \sqrt{1+\bar{u}^{\prime}\left(r^{2} / 4\right)^{2}} r^{2 n+1} \frac{d r}{r}=2^{2 n-1} \sigma_{2 n-1} \int_{0}^{R^{2} / 4} \sqrt{1+\bar{u}^{\prime}(s)^{2}} s^{(Q-3) / 2} d s .
\end{aligned}
$$

Formula (3.40), in which $\beta=-R / 2$, gives

$$
\sqrt{1+\bar{u}^{\prime}(s)^{2}}=\frac{R}{\sqrt{R^{2}-4 s}} .
$$

Inserting this equation in the above integral we obtain

$$
\int_{\Omega} \sqrt{\left|\nabla_{z} u_{o}\right|^{2}+\frac{|z|^{2}}{4}+<\nabla_{z} u_{o}, z^{\perp}>} d z=2^{2 n-1} \sigma_{2 n-1} R \int_{0}^{R^{2} / 4} s^{\frac{Q-4}{2}} \sqrt{\frac{s}{R^{2}-4 s}} d s .
$$

We notice that the last integral above is similar to the one in (3.49). Proceeding as in the last part of the proof of Theorem 3.18 we finally reach the conclusion.

At this point, recalling that (3.29) represents the Euler-Lagrange equation of the unconstrained functional (3.28), and keeping (3.27) in mind, if we combine Theorem 3.18 with Corollary 3.19, and take Remark 3.15] into account, we obtain the following result.

Theorem 3.21. Let $J$ and $G$ be the functionals

$$
J[u]=\int_{\operatorname{supp}(u)} f\left(z, \nabla_{z} u(z)\right) d z, \quad G[u]=\int_{\operatorname{supp}(u)} g(u) d z,
$$


where $f$ and $g$ are defined in (3.27). Given $V>0$, there exists $R=R(V)>0$ (see (3.43) such that the function $u_{o}=u_{R}$ in (3.45) is a critical point on $\mathcal{D}$ of the functional $J[u]$ subject to the constraint $G[u]=\frac{V}{2}$. This follows from the fact that $u_{o}$ is a critical point on $\mathcal{D}$ of the unconstrained functional $\mathcal{J}[u]$ in (3.28).

Our next objective is to prove that the function $u_{o}$ in (3.45) is the unique minimizer of the variational problem (3.25) on $\mathcal{D}$. We will need some basic facts from Calculus of Variations, which we now recall.

Definition 3.22. In the general perspective of Remark 3.13, let $\mathcal{V}$ be a normed vector space, and $\mathcal{D} \subset \mathcal{V}$ be a subset for which $\mathcal{A}_{\mathcal{D}} \neq \varnothing$. Given a functional $\mathcal{F}: \mathcal{D} \rightarrow \mathbb{R}, u \in \mathcal{D}$ and $\phi \in \mathcal{A}_{\mathcal{D}}$, one calls

$$
\delta \mathcal{F}(u ; \phi) \stackrel{\text { def }}{=} \lim _{\epsilon \rightarrow 0^{+}} \frac{\mathcal{F}[u+\epsilon \phi]-\mathcal{F}[u]}{\epsilon}
$$

the Gâteaux derivative of $\mathcal{F}$ at $u$ in the direction $\phi$ if the limit exists.

Definition 3.23. Let $\mathcal{V}$ be a normed vector space, and $\mathcal{D} \subset \mathcal{V}$ be a subset for which $\mathcal{A}_{\mathcal{D}} \neq \varnothing$. Consider a functional $\mathcal{F}: \mathcal{D} \rightarrow \overline{\mathbb{R}} . \mathcal{F}$ is said to be convex over $\mathcal{D}$ if for every $u, \in \mathcal{D}$, and every $\phi \in \mathcal{A}_{\mathcal{D}}$

$$
\mathcal{F}[u+\phi]-\mathcal{F}[u] \geq \delta \mathcal{F}(u ; \phi) \quad \text { for all } u \in \mathcal{D}, \phi \in \mathcal{A}_{\mathcal{D}},
$$

whenever the right-hand side is defined. We say that $\mathcal{F}$ is strictly convex if strict inequality holds in the above inequality except when $\phi \equiv 0$.

We have the following

Theorem 3.24. Suppose $\mathcal{F}$ is strictly convex and proper over $\mathcal{D} \subset V$ (i.e., $\mathcal{F} \not \equiv \infty$ over $\mathcal{D}$ ), and suppose that $u_{o} \in \mathcal{D}$ is such that $\delta \mathcal{F}\left(u_{o} ; \phi\right)=0$ for all $\phi \in \mathcal{A}_{\mathcal{D}}$ (that is, $u_{o}$ is a critical point of the functional $\mathcal{F})$, then $u_{o}$ is the unique element in $\mathcal{D}$ satisfying

$$
\mathcal{F}\left[u_{o}\right]=\inf \{\mathcal{F}[v] \mid v \in \mathcal{D}\} .
$$

Proof. From Definition 3.23 we immediately infer that if $u \neq u_{o}$ then with $\phi=u-u_{o} \in \mathcal{A}_{\mathcal{D}}$ we have

$$
\mathcal{F}[u]-\mathcal{F}\left[u_{o}\right]=\mathcal{F}\left[u_{o}+\phi\right]-\mathcal{F}\left[u_{o}\right]>\delta \mathcal{F}\left(u_{o} ; \phi\right)=0 .
$$

This shows $\mathcal{F}[u]>\mathcal{F}\left[u_{o}\right]$. This also shows uniqueness of such an $u_{o}$.

Our next goal is to adapt the above results to the problem (3.25). For a $C^{1}$ bounded open set $\Omega \subset \mathbb{R}^{2 n}$, we consider the normed vector space $\mathcal{V}=C(\bar{\Omega})$ with subset $\mathcal{D}$, where $\mathcal{D}$ is as in (3.22) of Definition 3.11. Let $h=h(z, u, \vec{p})$ be a function $h: \bar{\Omega} \times \mathbb{R} \times \mathbb{R}^{n} \rightarrow \mathbb{R}$ such that for each $z \in \Omega, h(z, \cdot, \cdot) \in C^{1}\left(\Omega_{i}\right), i=1,2, \ldots, m$, where $\Omega=\Omega_{1} \cup \Omega_{2} \cup \cdots \cup \Omega_{m}$, and $\left|\overline{\Omega_{i}} \cap \overline{\Omega_{j}}\right|=0$ if $i \neq j$. We define $\mathcal{F}: \mathcal{V} \rightarrow \mathbb{R}$ to be

$$
\mathcal{F}[u] \stackrel{\text { def }}{=} \int_{\Omega} h(z, u(z), \nabla u(z)) d z .
$$


In this situation we see that $\mathcal{F}$ is Gâteaux differentiable and

$$
\delta \mathcal{F}(u ; \phi)=\int_{\Omega} h_{u}(z, u(z), \nabla u(z)) \phi(z)+<\nabla_{p} h(z, u(z), \nabla u(z)), \nabla \phi(z)>d z,
$$

where in the above $\langle\cdot, \cdot\rangle$ denotes the standard inner product on $\mathbb{R}^{2 n}$. One has the following sufficient condition for the (strict) convexity of $\mathcal{F}$.

Proposition 3.25. If for a.e. $z \in \Omega$, for all $v \in \mathbb{R}$ and $p \in \mathbb{R}^{2 n}$, the function $h$ in the definition of $\mathcal{F}$ satisfies

$$
h(z, u+v, p+q)-h(z, u, p)>h_{u}(z, u, p) v+<\nabla_{p} h(z, u, p), q>
$$

unless $v=0$ and $q=0$, then $\mathcal{F}$ is strictly convex. If in (3.52) the non strict inequality $\geq$ holds (regardless whether $v=0$ or $q=0$ or not), then the functional $\mathcal{F}$ is convex.

Proof. The proof of the $\geq$ part is a trivial modification of the following for the $>$ version. Let $u, \phi \in \mathcal{V}$ be such that $\phi$ does not vanish identically. Letting $v=\phi$ and $q=\nabla \phi$ in (3.52), and using (3.51), we obtain

$$
\begin{aligned}
\mathcal{F}[u+\phi]-\mathcal{F}[u] & =\int_{\Omega}\{h(z, u(z)+\phi(z), \nabla u(z)+\nabla \phi(z))-h(z, u(z), \nabla u(z))\} d z \\
& >\int_{\Omega}\left\{h_{u}(z, u(z), \nabla u(z)) \phi(z)+<\nabla_{p} h(z, u(z), \nabla u(z)), \nabla \phi(z)>\right\} d z \\
& =\delta \mathcal{F}(u ; \phi) .
\end{aligned}
$$

Now appealing to Definition 3.23 the conclusion follows.

We now give the proof of the existence and uniqueness part of Theorem 1.1.

Proof of Theorem 1.1 (existence and uniqueness part). We fix $V>0$ and consider the collection of all sets $E \in \mathcal{E}$ such that $V=|E|$. We want to show that the problem of minimizing $P_{H}\left(E ; \mathbb{H}^{n}\right)$ within this subclass admits a unique solution, and that the latter is given by (3.45), in which the parameter $R=R(V)$ has been chosen as in (3.43). According to condition (i) in the definition of the class $\mathcal{E}$, we have $V / 2=\left|E \cap \mathbb{H}_{+}^{n}\right|$. Still from assumption $(i)$, and in view of Theorem 3.10, it is enough to minimize $P_{H}\left(E ; \mathbb{H}_{+}^{n}\right)$. This is an important point. In fact, Theorem [3.10] states that, if $E$ is an isoperimetric set, i.e., if $E$ minimizes $P_{H}\left(\circ ; \mathbb{H}^{n}\right)$ under the constraint $|E|=V$, then

$$
P_{H}\left(E ; \mathbb{H}_{+}^{n}\right)=P_{H}\left(E ; \mathbb{H}_{-}^{n}\right) .
$$

This implies that the minimizer must be sought for within the class of sets $E \in \mathcal{E}$ such that $|E|=V$, and for which (3.53) holds.

By the method of Lagrange multipliers, this problem is converted, in turn, into that of minimizing the unconstrained functional $\mathcal{J}[u]$ defined by (3.28) over this class. We want to show that $\mathcal{J}$ is strictly convex.

By condition (ii) there exists $R>0$ and $u \in C(\bar{B}(0, R)) \cap C^{1}(B(0, R))$, with $u \geq 0$ and $u=0$ on $\partial B(0, R)$, such that $\partial E \cap \mathbb{H}_{+}^{n}$ is described by the graph of $u$. Here, the parameter $R>0$ is the 
one obtained in Theorem 3.21. First, we derive the functionals that correspond to $P_{H}\left(E ; \mathbb{H}^{n}\right)$, $|E|$ and the Euler-Lagrange equation associated to the functionals for sets $E \in \mathcal{E}$.

Let $\mathcal{J}$ be given by (3.28). To this purpose, we observe that from (3.51) and (3.27), one can easily see that if $\operatorname{supp}(u)=B(0, R)$,

$$
\delta \mathcal{J}(u ; \phi)=\int_{B(0, R)}\left\{\frac{<\nabla_{z} \phi, \nabla_{z} u+z^{\perp} / 2>}{\left|\nabla_{z} u+z^{\perp} / 2\right|}+\lambda \phi\right\} d z
$$

and that furthermore,

$$
\begin{aligned}
\mathcal{J}[u+\phi] & -\mathcal{J}[u] \\
& =\int_{B(0, R)}\left\{\left|\nabla_{z} u+z^{\perp} / 2+\nabla_{z} \phi\right|-\left|\nabla_{z} u+z^{\perp} / 2\right|+\lambda \phi\right\} d z \\
& =\int_{B(0, R)}\left\{\frac{2<\nabla_{z} \phi, \nabla_{z} u+z^{\perp} / 2>+\left|\nabla_{z} \phi\right|^{2}}{\left|\nabla_{z} u+z^{\perp} / 2\right|+\left|\nabla_{z} u+z^{\perp} / 2+\nabla_{z} \phi\right|}+\lambda \phi\right\} d z .
\end{aligned}
$$

For simplicity, we now let $\vec{\alpha}=\nabla_{z} u+z^{\perp} / 2$ and $\vec{q}=\nabla_{z} \phi$ in (3.55). We intend to show that

$$
\frac{2<\vec{q}, \vec{\alpha}>+|\vec{q}|^{2}}{|\vec{\alpha}|+|\vec{\alpha}+\vec{q}|} \geq \frac{<\vec{q}, \vec{\alpha}>}{|\vec{\alpha}|},
$$

with strict inequality unless $\phi \equiv 0$. This would imply

$$
\frac{2<\nabla_{z} \phi, \nabla_{z} u+z^{\perp} / 2>+\left|\nabla_{z} \phi\right|^{2}}{\left|\nabla_{z} u+z^{\perp} / 2\right|+\left|\nabla_{z} u+z^{\perp} / 2+\nabla_{z} \phi\right|} \geq \frac{<\nabla_{z} \phi, \nabla_{z} u+z^{\perp} / 2>}{\left|\nabla_{z} u+z^{\perp} / 2\right|}
$$

with strict inequality unless $\phi \equiv 0$, which would prove that $\mathcal{J}$ is strictly convex. The inequality (3.56) is easily seen to be equivalent to

$$
(|\vec{q}+\vec{\alpha}|-|\vec{\alpha}|)<\vec{q}, \vec{\alpha}>\leq|\vec{q}|^{2}|\vec{\alpha}|
$$

The latter inequality is an easy consequence of the Cauchy-Schwarz and triangle inequalities. Equality holds only when $\vec{q}=\overrightarrow{0}, \vec{\alpha}=\overrightarrow{0}$, or $\vec{q}=\rho \vec{\alpha}$ for some $\rho \in \mathbb{R}$. These conditions translate into

$$
\nabla_{z} \phi \equiv \overrightarrow{0}, \quad \nabla_{z} u+z^{\perp} / 2=0, \quad \text { and } \quad \nabla_{z} \phi=\rho\left(\nabla_{z} u+z^{\perp} / 2\right) .
$$

However, since $\left.\phi\right|_{\partial B(0, R)}=0$, one can see that these conditions cannot be fulfilled. unless $\phi \equiv 0$. In Theorem 3.21 we have established that the function $u_{R}$ (defined by the formula (3.45) ) satisfies

$$
\delta \mathcal{J}\left(u_{R}, \phi\right)=0
$$

for every $\phi$ with $\left.\phi\right|_{\partial B(0, R)}=0$. Now appealing to Theorem 3.24 we conclude that $u_{R}$ given by (3.45) is the unique minimizer to the variational problem (3.25) with $\Omega=B(0, R)$, and $R$ given by Theorem 3.18

We will now complete the proof of Theorem 1.1 by establishing two properties of the isoperimetric profiles, Propositions 3.26 and 3.27 below. First, we discuss their regularity. It suffices 
to consider the "normalized" isoperimetric profile $E_{o} \subset \mathbb{H}^{n}$ which provides the unique solution in Theorem 1.1. The characteristic locus of $E_{o}$ is given by the two points in $\mathbb{H}^{n}$

$$
\Sigma=\left\{\left(0,0, \pm \frac{\pi}{8}\right)\right\}
$$

Unlike its Euclidean counterpart, the hypersurface surface $\partial E_{o}$ is not $C^{\infty}$ at the characteristic points $\left(0,0, \pm \frac{\pi}{8}\right)$.

Proposition 3.26. The hypersurface $S_{o}=\partial E_{o} \in \mathbb{H}^{n}$ is $C^{2}$, but not $C^{3}$, near its characteristic locus $\Sigma$. However, $S_{o}$ is $C^{\infty}$ (in fact, real-analytic) away from $\Sigma$.

Proof. First, we show that near the characteristic points $\left(0,0, \pm \frac{\pi}{8}\right)$ the function $u_{o}(z)$ given by (1.5) is only of class $C^{2}$, but not of class $C^{3}$. To see this we let

$$
u_{1}(s)=\frac{\pi}{8}+\frac{s}{4} \sqrt{1-s^{2}}-\frac{1}{4} \sin ^{-1}(s), 0 \leq s \leq 1 .
$$

and note that $u_{o}(z)=u_{1}(|z|)$ for $0 \leq|z| \leq 1$. Therefore, the regularity of $u_{o}$ at $|z|=0$ is equivalent to verifying up to what order of derivatives $n$ one has

$$
\lim _{s \rightarrow 0^{+}} u_{+}^{(n)}(s)=\lim _{s \rightarrow 0^{-}} u_{-}^{(n)}(s)
$$

where $u_{+}(s)=u_{1}(s)$ and $u_{-}(s)=u_{1}(-s)$. It is easy to compute

$$
\begin{aligned}
& -u_{-}^{\prime}(s)=u_{+}^{\prime}(s)=-\frac{1}{2} \frac{s^{2}}{\sqrt{1-s^{2}}}, \quad-u_{-}^{\prime \prime}(s)=u_{+}^{\prime \prime}(s)=-\frac{1}{2} \frac{s\left(s^{2}-2\right)}{\left(s^{2}-1\right) \sqrt{1-s^{2}}}, \\
& -u_{-}^{(3)}(s)=u_{+}^{(3)}(s)=-\frac{1}{2} \frac{2+s^{2}}{\left(s^{2}-1\right)^{2} \sqrt{1-s^{2}}} .
\end{aligned}
$$

We clearly have

$$
\lim _{s \rightarrow 0^{-}} u_{-}^{(n)}=\lim _{s \rightarrow 0^{+}} u_{+}^{(n)} \quad \text { for } n=0,1,2 \quad \text { whereas } \lim _{s \rightarrow 0^{-}} u_{-}^{(3)}=1 \text { and } \lim _{s \rightarrow 0^{+}} u_{+}^{(3)}=-1 .
$$

This shows the function $t=u_{o}(z)$ is only $C^{2}$, but not $C^{3}$, near $z=0$. Next, we investigate the regularity of $\partial E_{o}$ near $|z|=1$, that is, at the points where the upper and lower branches that form $\partial E_{o}$ meet. To this end, we observe that $\partial E_{o}$ can also be generated by rotating around the $t$-axis the curve in the $\left(x_{1}, t\right)$-plane whose trace is

$$
\left\{\left(x_{1}, t\right) \mid t^{2}=u_{1}\left(x_{1}\right)^{2}, \quad 0 \leq x_{1} \leq 1\right\} .
$$

It suffices to show that this curve is smooth $\left(C^{\infty}\right)$ across the $x_{1}$ axis. To this end we compute the derivatives of $u_{1}$. It is easy to see by induction that for $n \geq 3$

$$
u_{1}^{(n)}\left(x_{1}\right)=(-1)^{n} C_{n} \frac{P_{n-1}\left(x_{1}\right)}{\left(x_{1}^{2}-1\right)^{n-1} \sqrt{1-x_{1}^{2}}},
$$

where $C_{n}>0$ is a constant depending only on $n$, and $P_{n-1}\left(x_{1}\right)$ is a polynomial in $x_{1}$ of degree $n-2$. The $n$-th derivatives of the function $-u_{1}\left(x_{1}\right)$ clearly takes the same form, but with a negative sign. Letting $s \rightarrow 1^{-}$in (3.58) we see that

$$
\frac{d^{n}}{d x_{1}^{n}} u_{1}, \frac{d^{n}}{d x_{1}^{n}}\left(-u_{1}\right) \longrightarrow \pm \infty, \quad \text { (depending on whether } n \text { is odd or even) . }
$$

This implies that the curve with equation $t^{2}=u_{1}\left(x_{1}\right)^{2}$ is smooth across the $x_{1}$-axis. 
Proposition 3.27. Suppose $E \in \mathcal{E}$ is a critical point of the $H$-perimeter subject to the constraint $|E|=$ const. If, in addition, $E$ is of class $C^{2}$, then $S=\partial E$ has constant $H$-mean curvature. In particular, the isoperimetric set $E_{o}$ found in Theorem 1.1 is a set of constant positive $H$-mean curvature.

Proof. Let $E \in \mathcal{E}$ be given and let $u$ be the function describing $\partial E$ in $\mathbb{H}_{+}^{n}$. To prove that $\partial E$ has constant $H$-mean curvature we could appeal to Remark 3.16. Instead, we proceed directly as follows. We recall that $u(z)=\bar{u}\left(|z|^{2} / 4\right)$ for some $C^{2}$ function $\bar{u}$, and the assumptions that $E$ is a critical point of the $H$-perimeter means that $\bar{u}$ satisfies (3.34). From the discussion in the proof of Theorem [3.17, the left hand side of (3.34) (that is the Euler-Lagrange equation) becomes

$$
r F^{\prime}(r)+(Q-2) F(r)
$$

where $F(r)$ is given by (3.35). A simple computation gives

$$
F^{\prime}(r)=\frac{r^{2} \bar{u}^{\prime \prime}\left(r^{2} / 4\right)-2 \bar{u}^{\prime}\left(r^{2} / 4\right)\left(1+\bar{u}^{\prime}\left(r^{2} / 4\right)^{2}\right)}{2 r^{2}\left(1+\bar{u}^{\prime}\left(r^{2} / 4\right)^{2}\right)^{\frac{3}{2}}},
$$

and therefore we have

$$
r F^{\prime}(r)+(Q-2) F(r)=\frac{2(Q-3) \bar{u}^{\prime}\left(r^{2} / 4\right)\left(1+\bar{u}^{\prime}\left(r^{2} / 4\right)^{2}\right)+r^{2} \bar{u}^{\prime \prime}\left(r^{2} / 4\right)}{2 r\left(1+\bar{u}^{\prime}\left(r^{2} / 4\right)^{2}\right)^{\frac{3}{2}}} .
$$

Rewriting the Euler-Lagrange equation (3.34) for such functions $u$ (or $\bar{u}$ ) we have

$$
\frac{2(Q-3) \bar{u}^{\prime}\left(r^{2} / 4\right)\left(1+\bar{u}^{\prime}\left(r^{2} / 4\right)^{2}\right)+r^{2} \bar{u}^{\prime \prime}\left(r^{2} / 4\right)}{2 r\left(1+\bar{u}^{\prime}\left(r^{2} / 4\right)^{2}\right)^{\frac{3}{2}}}=\lambda
$$

where $\lambda$ is of course a constant. We make a change of notation by letting $s=r^{2} / 4$ in (3.60), we found

$$
\frac{(Q-3) \bar{u}^{\prime}(s)\left(1+\bar{u}^{\prime}(s)^{2}\right)+2 s \bar{u}^{\prime \prime}(s)}{2 \sqrt{s}\left(1+\bar{u}^{\prime}(s)^{2}\right)^{\frac{3}{2}}}=\lambda .
$$

Comparing (3.61) with (3.14), we infer that the $H$-mean curvature of such surfaces is

$$
\mathcal{H}=-\frac{(Q-3) \bar{u}^{\prime}(s)\left(1+\bar{u}^{\prime}(s)^{2}\right)+2 s \bar{u}^{\prime \prime}(s)}{2 \sqrt{s}\left(1+\bar{u}^{\prime}(s)^{2}\right)^{\frac{3}{2}}}=-\lambda .
$$

If the set $E_{o}$ is described by $u_{R}(z)$, where $u_{R}(z)$ is given by (3.45), then from (3.44) in Theorem 3.18 we conclude that the $H$-mean curvature of $E_{o}$ is given by

$$
\mathcal{H}=\frac{Q-2}{R} .
$$

This completes proof of Theorem 1.1.

Proof of Theorem 1.2. We have already established the restricted isoperimetric inequality. Furthermore, the invariance of the isoperimetric quotient with respect to the group translations and dilations is a consequence of Propositions 2.11 and 2.12. We are left with the computation of the constant $C_{Q}$. To this end, we use the set $E_{R}$ described by $u_{o}$. We note that the integrals 
(3.47) and (3.50) give $\left|E_{R}\right| / 2$ and $P_{H}\left(E ; \mathbb{H}_{+}^{n}\right)$ respectively, and therefore after some elementary simplifications we obtain

$$
C_{Q}=\frac{\left|E_{R}\right|^{\frac{Q-1}{Q}}}{P_{H}\left(E_{R} ; \mathbb{H}^{n}\right)}=\frac{(Q-1) \Gamma\left(\frac{Q}{2}\right)^{\frac{2}{Q}}}{Q^{\frac{Q-1}{Q}}(Q-2) \Gamma\left(\frac{Q+1}{2}\right)^{\frac{1}{Q}} \pi^{\frac{Q-1}{2 Q}}} .
$$

This completes the proof.

\section{REFERENCES}

[A] A. D. Alexandrov, A characteristic property of spheres, Ann. Mat. Pura Appl., (4) 58 1962, 303-315.

[B] Z. M. Balogh, Size of characteristic sets and functions with prescribed gradients, J. Reine Angew. Math., $\mathbf{5 6 4} 2003,63-83$.

[Be] A. Bellaïche, The tangent space in sub-Riemannian geometry. Sub-Riemannian geometry,, Progr. Math., 144 (1996), Birkhäuser, 1-78.

[BM] M. Biroli \& U. Mosco, Sobolev and isoperimetric inequalities for Dirichlet forms on homogeneous spaces, Pot. Anal., 4 (1995), 311-324.

[BC] M. Bonk \& L. Capogna, Mean Curvature flow and the isoperimetric profile of the Heisenberg group, preprint, 2005.

[CDG] L. Capogna, D. Danielli \& N. Garofalo, The geometric Sobolev embedding for vector fields and the isoperimetric inequality, Comm. Anal. and Geom., 2 (1994), 201-215.

[CG] L. Capogna \& N. Garofalo, Ahlfors type estimates for perimeter measures in Carnot-Carathéodory spaces, J. Geom. Anal., to appear.

[Ca] C. Carathéodory, Untersuchungen über die Grundlangen der Thermodynamik, Math. Ann., 67 (1909), 355386.

[CH] J.H.Cheng \& J.F. Hwang, Properly embedded and immersed minimal surfaces in the Heisenberg group, Bull. Austral. Math. Soc., 70 (2004), no. 3, 507-520.

[CHMY] J.H. Cheng, J. F. Hwang, A. Malchiodi \& P. Yang, Minimal surfaces in pseudohermitian geometry and the Bernstein problem in the Heisenberg group, revised version 2004, Ann. Sc. Norm. Sup. Pisa, 1 (2005), 129-177.

[Ch] W. L. Chow, Über Systeme von linearen partiellen Differentialgleichungen erster Ordnung, Math. Annalen, 117 (1939), 98-105.

[CS] T. Coulhon \& L. Saloff-Coste, Isopérimétrie pour les groupes et les variétés, Rev. Mat. Iberoamericana, 9 (1993), 293-314.

[DGN1] D. Danielli, N. Garofalo \& D. M. Nhieu, Trace inequalities for Carnot-Carathéodory spaces and applications, Ann. Sc. Norm. Sup. Pisa, Cl. Sci. (4), 2,27 (1998), 195-252.

[DGN2] _ Non-doubling Ahlfors measures, Perimeter measures, and the characterization of the trace spaces of Sobolev functions in Carnot-Carathéodory spaces, preprint, 2002.

[DGN3] D. Danielli, N. Garofalo \& D. M. Nhieu, Notions of convexity in Carnot groups, Comm. Anal. and Geom., 11, no.2, (2003), 263-341.

[DGN4] - Minimal surfaces in Carnot groups, preprint, 2005.

[DG1] E. De Giorgi, Su una teoria generale della misura $(r-1)$-dimensionale in uno spazio a $r$ dimensioni, Ann. Mat. Pura Appl., 36 (1954), 191-213.

[DG2] - Nuovi teoremi relativi alla misura $(r-1)$-dimensionale in uno spazio a $r$ dimensioni, Ric. Mat., 4 (1955), 95-113.

[DG3] _ Sulla proprietà isoperimetrica dell'ipersfera, nella classe degli insiemi aventi frontiera orientata di misura finita, (Italian) Atti Accad. Naz. Lincei. Mem. Cl. Sci. Fis. Mat. Nat. Sez. I (8), 5 1958 33-44.

[DCP] E. De Giorgi, F. Colombini \& L. C. Piccinini, Frontiere orientate di misura minima e questioni collegate, Sc. Norm. Sup. Pisa, Cl. Scienze, Quaderni, 1972.

[De1] M. Derridj, Un probléme aux limites pour une classe d'opérateurs du second ordre hypoelliptiques, Ann. Inst. Fourier, Grenoble, 21, 4 (1971), 99-148.

[De2] _ Sur un théorème de traces, Ann. Inst. Fourier, Grenoble, 22, 2 (1972), 73-83. 
[E1] P. Eberlein, Geometry of 2-step nilpotent groups with a left invariant metric, Ann. Sci. ?cole Norm. Sup. (4) 27 (1994), no. 5, 611-660.

[E2] - Geometry of 2-step nilpotent groups with a left invariant metric. II, Trans. Amer. Math. Soc., 343 (1994), no. 2, 805-828.

[E3] - Geometry of nonpositively curved manifolds, Chicago Lectures in Mathematics. University of Chicago Press, Chicago, IL, 1996.

[Fe] H. Federer, Geometric Measure Theory, Springer, 1969.

[FMP] C. B. Figueroa, F. Mercuri \& R. H. L. Pedrosa, Invariant surfaces of the Heisenberg groups, Ann. Mat. Pura Appl. (4), 177 (1999), 173-194.

[FR] W. H. Fleming \& R. Rishel, An integral formula for total gradient variation, Arch. Math., 11 (1960), 218-222.

[F1] G. B. Folland, A fundamental solution for a subelliptic operator, Bull. Amer. Math. Soc., 79 (1973), 373-376.

[F2] _ Subelliptic estimates and function spaces on nilpotent Lie groups, Ark. Math., 13 (1975), $161-207$.

[F3] _ Harmonic Analysis in Phase Space, Ann. Math. Studies, Princeton Univ. Press, 1989.

[FGW] B. Franchi, S. Gallot \& R. Wheeden, Sobolev and isoperimetric inequalities for degenerate metrics, Math. Ann., 300 (1994), 557-571.

[FSS1] B. Franchi, R. Serapioni \& F. Serra Cassano, Meyers-Serrin type theorems and relaxation of variational integrals depending on vector fields. Houston J. Math. 22 (1996), no. 4, 859-890.

[FSS2] _ Rectifiability and perimeter in the Heisenberg group, Math. Ann., 321 (2001) 3, 479-531.

[FSS3] _ On the structure of finite perimeter sets in step 2 Carnot groups, J. Geom. Anal., 13 (2003), no. $3,421-466$.

[FSS4] _ Regular hypersurfaces, intrinsic perimeter and implicit function theorem in Carnot groups, Comm. Anal. Geom., 11 (2003), no. 5, 909-944.

[GN] N. Garofalo \& D. M. Nhieu, Isoperimetric and Sobolev inequalities for Carnot-Carathéodory spaces and the existence of minimal surfaces, Comm. Pure Appl. Math., 49 (1996), 1081-1144.

[GP] N. Garofalo \& S. D. Pauls, The Bernstein problem in the Heisenberg group, preprint, 2004.

[GH] M. Giaquinta \& S. Hildebrandt, Calculus of variations, I \& II. Grundlehren der Mathematischen Wissenschaften [Fundamental Principles of Mathematical Sciences], 310, 311. Springer-Verlag, Berlin, 1996.

[Gro1] M. Gromov, Carnot-Carathéodory spaces seen from within, in Sub-Riemannian Geometry, Progress in Mathematics, vol. 144, edited by André Bellä̈che \& Jean-Jacques Risler, Birkhäuser, 1996.

[Gro2] _ Metric Structures for Riemannian and Non-Riemannian Spaces, Ed. by J. LaFontaine and P. Pansu, Birkhäuser, 1998.

[HP] R. K. Hladky \& S. D. Pauls, Constant mean curvature surfaces in sub-Riemannian geometry, preprint, 2005.

[H] H. Hörmander, Hypoelliptic second-order differential equations, Acta Math., 119 (1967), 147-171.

[LM] G. P. Leonardi \& S. Masnou, On the isoperimetric problem in the Heisenberg group $\mathbb{H}^{n}$, Ann. Mat. Pura Appl., (4) 184 (2005), no. 4, 533-553.

[LR] G. P. Leonardi \& S. Rigot, Isoperimetric sets on Carnot groups, Houston J. Math., 29 (2003), no. 3, 609-637.

[Ma] V. Magnani, Characteristic points, rectifiability and perimeter measure on stratified groups, preprint, 2004.

[MaSC] P. Maheux \& L. Saloff-Coste, Analyse sur les boules d'un opérateur sous-elliptique, Math. Ann., 303 (1995), 713-740.

[MM] U. Massari \& M. Miranda, Minimal Surfaces of Codimension One, Math. Studies 91, North-Holland, 1984.

[Mo1] R. Monti, Some properties of Carnot-Carathodory balls in the Heisenberg group, Atti Accad. Naz. Lincei Cl. Sci. Fis. Mat. Natur. Rend. Lincei (9), Mat. Appl.textbf11 (2000), no. 3, 155-167 (2001).

[Mo2] - Brunn-Minkowski and isoperimetric inequality in the Heisenberg group, Ann. Acad. Sci. Fenn. Math., 28 (2003), no. 1, 99-109.

[MoM] R. Monti \& D. Morbidelli, Isoperimetric inequality in the Grushin plane, J. Geom. Anal., 14 (2004), no. 2, 355-368.

[P1] P. Pansu, Une inégalité isopérimétrique sur le groupe de Heisenberg, C. R. Acad. Sci. Paris Sér. I Math., 295 (1982), no. 2, 127-130.

[P2] P. Pansu, An isoperimetric inequality on the Heisenberg group, Conference on differential geometry on homogeneous spaces (Torino, 1983). Rend. Sem. Mat. Univ. Politec. Torino 1983, Special Issue, 159-174 (1984).

[Pa] S. Pauls, Minimal surfaces in the Heisenberg group, Geom. Dedicata, 104 (2004), 201-231.

[RR] M. Ritorè \& C. Rosales, Rotationally invariant hypersurfaces with constant mean curvature in the Heisenberg group $\mathbb{H}^{n}$, preprint, April 2005.

[STh] I. M. Singer \& J. A. Thorpe Lecture notes on elementary topology and geometry, Scott-Foresman, Glenview, Illinois, 1967.

[Tr] J. L. Troutman, Variational Calculus with Elementary Convexity, Springer-Verlag, 1983.

[St] E. M. Stein, Harmonic Analysis: Real Variable Methods, Orthogonality and Oscillatory Integrals, Princeton Univ. Press, (1993). 
[To] P. Tomter, Constant mean curvature surfaces in the Heisenberg group, Differential Geometry: Partial Differential Equations of Manifolds (Los Angeles, CA, 1990), p.485-495, Amer. Math. Soc., Providence, RI, 1993.

[Va1] N. Th. Varopoulos, Analysis on nilpotent groups, J. Funct. Anal., 66 (1986), no.3, 406-431.

[Va2] _ Analysis on Lie groups, J. Funct. Anal., 67 (1988), no.2, 346-410.

[VSC] N. Th. Varopoulos, L. Saloff-Coste \& T. Coulhon, Analysis and Geometry on Groups, Cambridge U. Press, 1992.

Department of Mathematics, Purdue University, West Lafayette, IN 47907

E-mail address, Donatella Danielli: danielli@math.purdue.edu

Department of Mathematics, Purdue University, West Lafayette, IN 47907

E-mail address, Nicola Garofalo: garofalo@math.purdue.edu

Dip. Metodi e Modelli Matematici per le Scienze Applicate, Univ. Padova, 35100 Padova, Italy

E-mail address, Nicola Garofalo: garofalo@math.purdue.edu

Department of Mathematics, Georgetown University, Washington DC 20057-1233

E-mail address, Duy-Minh Nhieu: nhieu@math.georgetown.edu 